This item was submitted to Loughborough's Research Repository by the author.

Items in Figshare are protected by copyright, with all rights reserved, unless otherwise indicated.

\title{
A network control system for hydro plants to counteract the nonsynchronous generation integration
}

PLEASE CITE THE PUBLISHED VERSION

https://doi.org/10.1016/j.jijepes.2018.08.020

PUBLISHER

(C) Elsevier

VERSION

AM (Accepted Manuscript)

\section{PUBLISHER STATEMENT}

This paper was accepted for publication in the journal International Journal of Electrical Power and Energy Systems and the definitive published version is available at https://doi.org/10.1016/j.ijepes.2018.08.020.

\section{LICENCE}

CC BY-NC-ND 4.0

\section{REPOSITORY RECORD}

Chamorro, Harold R., Andres C. Sanchez, Andres Pantoja, Ivan Zelinka, Francisco M. Gonzalez-Longatt, and Vijay K. Sood. 2019. "A Network Control System for Hydro Plants to Counteract the Nonsynchronous Generation Integration”. figshare. https://hdl.handle.net/2134/34770. 


\title{
A Network Control System for Hydro Plants to Counteract the Non-Synchronous Generation Integration
}

\author{
Harold R. Chamorro ${ }^{\mathrm{a}, 1}$, Andres C. Sanchez ${ }^{\mathrm{b}}$, Andres Pantoja ${ }^{\mathrm{c}}$, Ivan Zelinka ${ }^{\mathrm{d}}$, Francisco Gonzalez-Longatt ${ }^{\mathrm{e}}$, \\ Vijay K. Sood \\ ${ }^{a}$ Department of Electric Power Systems, KTH Royal Institute of Technology, Tekninkringen 33, 10044 Stockholm, Sweden \\ ${ }^{b}$ Universidad de los Andes, Bogota, Colombia \\ ${ }^{c}$ Universidad de Narino, Narino, Colombia \\ ${ }^{d}$ VSB Technical University of Ostrava, Ostrava, Czech Republic \\ ${ }^{e}$ Loughborough University, Leicestershire, United Kingdom \\ ${ }^{f}$ Department of Electrical and Computer Engineering of the University of Ontario, Institute of Technology, Ontario, Canada
}

\begin{abstract}
Sweden is a country with abundant hydro power and has expectations to include more renewable energy sources, namely from wind power, into its electrical system. Currently, in order to improve the frequency response requirements of its electrical system, the country is considering upgrading its hydro-governors. This effort is part of maintaining the system frequency and reaction within their limits following any disturbance events. To partially compensate for increased frequency fluctuations due to an increased share of renewables on its system, the frequency response of hydro-governors should be improved. This paper proposes an innovative network control system, through a supplementary control, for the improvement of the hydro-governor's action. This supplementary control allows having more flexibility over the control action and improves the primary frequency control, and thereby the overall system frequency response. The proposed supplementary control, based on an evolutionary game theory strategy, uses remote measurements and a hierarchical dynamic adjustment of the control. Additionally, in order to guarantee an optimal response, a Simulated Annealing algorithm is combined with the supplementary control. This paper illustrates the analysis and design of the proposed methodology, and is tested on two power systems models: (i) an aggregated model that represents the frequency response of Sweden, Norway and Finland, and (ii) The Nordic 32 test system.
\end{abstract}

Keywords: Hydro-Governors, Frequency Control, Primary Control, Replicator Dynamics, Non-Synchronous Generation, Wind Power, Simulated Annealing Algorithm

\section{Introduction}

Concerns about global warming has motivated governmental agencies the world over to employ more friendly environmental policies. The current European energy goals set for 2030 and 2050 to enhance green power are a driving factor in the development and integration of more renewable resources into the grid [1]. Consequently, technical challenges in the power generation system have emerged due to the rapid development and integration of renewable power [2].

The renewable power interconnection has been possible due to the use of high-power electronics converters as a control interface of the power to be supplied to the grid. One of the challenges of the large integration of Renewable Energy Sources (RES) is the inertia reduction in the system which has resulted in a larger deviation from the nominal system frequency under a disturbance [3]. Thus, additional controller options are needed in the frequency process restoration [4].

\footnotetext{
*corresponding author, E-mail address:hrcv@kth.se,hr.chamo@ieee.org
}

Email address: hrcvakth. se (Harold R. Chamorro) 
One of the aspects that needs consideration is the maintenance of the frequency stability boundaries in the system [5], and adequate response in order to avoid undesirable events like outages or blackouts [6]. Potentially, hydrodominated systems can counteract the lack of inertia by improving the hydro-governors involved.

The hydro turbine governor is a system that regulates the inlet water into a turbine, which in turn rotates the generator to produce electricity. In order to maintain a required generated frequency at the reference value $(50$ or 60 $\mathrm{Hz}$ ), the turbine speed of rotation must be kept constant [7], [8]. The turbine governor receives information on the current rotational speed of the turbine and adjusts the water flow to maintain the speed at the correct level [9], [10].

The Nordic power system is mainly a hydro dominated system and frequency control are predominately performed by hydropower plants. Many of the governors currently in use in Sweden are of an older generation and purely mechanical design. While effective, these suffer from mechanical wear due to ageing. As these units reach the end of their working life, replacement is essential. At the present moment in time, the replacement of choice is a Proportional-Integral/Derivative $(P I / D)$ controller adapted to function as the governor for a turbine [11], [12].

A faster response of the governor in reacting and returning the system frequency close to the nominal values when subjected to a disturbance is desired.

Several proposals have been studied in order to enhanced the hydro-governors response. A hydro governing system based on fuzzy logic is presented in [13], where it is compared to a PID governor showing an improvement in the settling time and the overshoot. Another fuzzy proposal is compared to slide mode control for the performance improvement of a hydro governing system in [14].

In [15], a fuzzy $P I+D$ control structure is designed showing the possibility of including the derivative term as an extra signal involved in the hydro-governor control.

Authors in [16], proposed a robust control based on a high gain observer as an adjustable parameter for obtaining an adequate dynamic response under disturbances. A decentralized control signal for hydro governors has been designed using $H_{\infty}$ control in [17], showing the speed of response during different disturbances.

By using a feedback linear approach, the authors in [18], aimed to design a governor to deal with the transient stability and to damp the oscillations in the system. The authors in [19], present a robust control design for hydrogovernors based on additional inner states feedback signals and it is compared to traditional PI and PID architectures.

Analytical controllers such as the ones proposed in [16] and [19] might require a new design in case of a necessary modification in the system. In contrast, a fuzzy approach such as [13] and [15], could adapt themselves under a larger operation range.

In [20], an isolated system which includes a hydro governor and a wind turbine generation system is studied; the influence of both generations on the frequency under load changes is shown. A frequency control design framework for hydro power is proposed in [21], for counteracting the influence of the wind integration and reducing the frequency deviation.

In [22], the primary frequency control of hydro-dominated power system which integrates wind power is studied. It is focused on the pitch control and the frequency support from the turbine. A similar approach is given in [23], where a control method is developed to enhance the frequency capability including energy storage.

As a partial improvement for the integration of large renewable integration, several authors have proposed the application of the so-called virtual inertia. Authors in [24] added the derivative control in multi-area power systems.

Another example in the literature using the virtual inertia is given in [25]. It uses a similar approach of [24] with the application of control layers. Additionally a dynamic security constraint assessment is proposed by the power generation changes versus the droop coefficients.

In [26], a synchronous generation emulation for VSC stations is presented. Current and droop controllers are improved for achieving the frequency regulation under several scenarios.

In this context, a virtual inertia emulation is presented in [27], where linearized models are used for the frequency response modeling. Moreover, a maximum power tracking point method is applied to reduced the frequency fluctuations and operate under real wind conditions.

Another contribution perspective [28], where also there is a generation mix, presents the fusion of droop control and a pitch control, for improving the system frequency and reducing the need for reserves. A distributed Model Predictive Control is proposed in [29] for the frequency improvement including the coordination optimization strategy between the aggregated hydro-dominated systems and the wind power plant.

At the same time, the transformation of the power systems into a Cyber-Physical Systems (CPSs), where remote measurements and control interactions are combined, have brought opportunities to propose new control architectures 
for solving common goals in the systems [30], [31].

CPSs have been used widely in several power systems applications such as distributed automatic generation control [32], microgrids management [33], microgrids secondary/primary control [34], distributed Power System Stabilizers (PSS) [35], adaptive power flow control [36], distributed power injection in low-inertia power systems [37], distributed dispatch [38], and distributed active power control [39].

Under the framework of CPS and Multi-Agent System (MAS), several protocols have been deployed. Depending on the application, MAS can be classified or used as: decision making, commitment-based, coalition-forming, negotiation, and resource allocation [40]. Distributive controllers intended to cooperate to reach or improve certain common goal such as the frequency response improvement is the motivation of this document. Consensus or leader-follower protocols have shown to be a potential tool for the frequency control regulation (secondary control) in power systems reaching a distributive convergence to the nominal frequency [41].

Another MAS protocol is Replicator Dynamics (RDs) which belongs to the Evolutionary Game Theory (EGT). RDs is inspired by natural selection and uses a simple population dynamics that shows how individual or populations change their strategy over time based on payoff functions [42]. RD have been used in several MAS applications with successful results [38], [43].

RDs protocol has the property of maximizing the social welfare for group actions and plants. In the case of the governors, RD not only looks for the best response of one individual, but for the group as well, obtaining the equilibrium where all the derivative actions reach the best group action. Additionally, having an initial optimal value obtained from the SAA guarantees an optimal response.

As a remedial action in the primary frequency control performance due to the increasing non-synchronous generation and response enhancement, this paper proposes a control network paradigm using remote and local information in the hydro-governors through a supplementary signal. Our proposal aims to do a dynamic control distribution, in each of the $\mathrm{N}$-systems conformed by the hydro-governors involved, and optimize the frequency response in the system. For the latest, the optimal frequency response, it applies a novel procedure based on an evolutionary game theory, i.e., the RD, acting as a centralised controller [44], [38]. The design constraints are studied including the population game strategy. The applied method is compared to the Simulated Annealing algorithm (SAA) for obtaining the optimal local controllers in the system.

The paper is organized as follows: in Section 2 the theoretical preliminaries of graph theory and RD are presented. Then section 3 briefly presents notation and the application to the stated problem. In section 4, the power system preliminaries, the frequency power response, and the modeling of the system are introduced. Section 5 presents the control architecture, the measurement and the performance metrics, and the RD function proposed. Section 6 presents the simulation results considering the two systems, the aggregated model and the Nordic 32 test system. In the latter, a gradual inclusion of non-synchronous generation is included in order to observe the impact on the system frequency control, and the improvement by the presented method is shown. Finally, the conclusions and future work are given.

\section{Preliminaries}

\subsection{Information Graph Theory}

Consider a graph $\mathcal{G}=(v, \varepsilon)$ consisting of a set of vertices and edges $v=(1,2, \ldots, n)$. Nodes are adjacent if an edge/link exists between them. Based on this definition, a matrix called adjacency matrix $\mathcal{A}$ that shows the adjacency between the nodes in the graph $\mathcal{G}$ can be defined. The distance between two nodes i.e. $d(i, j)$ is the shortest path with least number of links that connect nodes $i$ and $j$. The degree matrix $\mathcal{D}$ with the elements $d_{i}$ of is a diagonal matrix which elements are the cardinality of agent $i$ neighbor set. The Laplacian matrix $\mathcal{L}$ is equal to the difference between degree matrix and adjacency matrix $(\mathcal{L}=\mathcal{D}-\mathcal{A})$. For a connected graph, Laplacian matrix $\mathcal{L}$ has exactly one zero eigenvalue and the eigenvalues increases by the order. The second smallest $\lambda_{2}(\mathcal{G})$ eigenvalue of $\mathcal{L}$ shows how well the graph is connected. Therefore, it is also known as the algebraic connectivity.

\subsection{Replicator Dynamics and Multi-Agent Systems}

The RD were introduced originally by Taylor and Jonker in [42]. RD is a selection mechanism based on population behavioral trends of limited individuals, and it has been used for optimization decomposition into several small-scale targets, and assign them to multiple homogeneous agents [45]. RD describes the evolution of the distribution of a 
normalized population of many players who have the same set of possible pure strategies $Q$, according to benefit provided by those strategies. Each player chooses a strategy, an earn and payoff depending on its strategy and the strategy distribution in the population. The generalized dynamics is formulated in continuous time as:

$$
\dot{x}_{i}=\beta x_{i}\left(f_{i}-\bar{f}\right)
$$

where $x_{i}$ is the state vector of portions of the population following the $i_{t h}$ strategy, for all $i=1, \ldots, q, f$ is the payoff function for strategy, which is a fitness function in behavioural ecology [46], [47], and $\bar{f}$ is the weighted average payoff. The function $\beta>0$ can be understood as an incentive for the rate of growth. An equilibrium is reached when $f=\bar{f}$. $\beta>0$ is a constant that ensures that $f_{i}$ is always positive ( $\beta$ is added for consistency with the biological counterpart of the proposed analogy) (to avoid numerical errors in the solution of differential equations). The weighted average payoff is expressed as:

$$
\bar{f}=\frac{1}{P} \sum_{j=1}^{N} x_{j} f_{j}
$$

where $P=\sum_{j=1}^{N} x_{i}$ is the total population. The fitness of each individual changes proportionally to the network reproduction rate of the population.

Figure 1 shows the network control system based on RD interacting with the topology agents. In the figure, two layers can be seen: the upper one represents the agents interaction, while the lower one depicts the power system topology. For the particular case, the primary control enhancement, the lower layer represents the governors distributively located in the power systems. The agents receive and aggregate the information and follow the RD protocol in order to distribute dynamically the controllers action suitable for each governor and for the group, achieving the most appropriate primary frequency response.

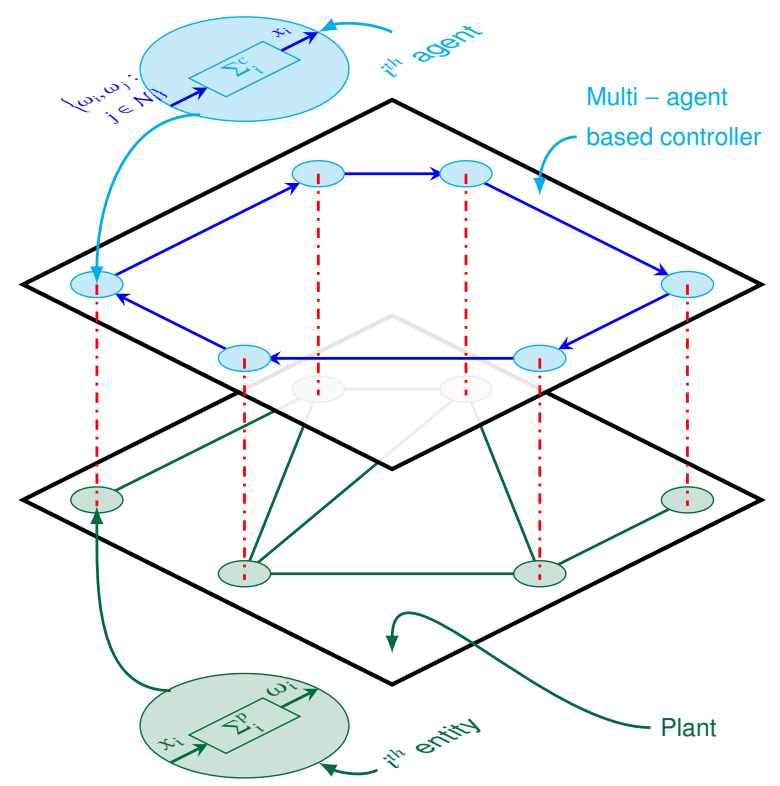

Figure 1: Large-scale System Controlled by a Network of Agents

\subsection{Optimization Properties of the RD}

Solving optimization problem by RD refers to dividing a global optimization target to be achieved into multiple targets between agents. Based on RD protocol and the sub-target, agents find optimal results in the evolution process of behavior choice. Evolutionary game-based approaches have been applied to multi-objective optimization problems in order to select weights of the objective functions and the best Pareto solution under some performance metrics [43].

According to the principle of RD protocol, the objective function can be adjusted, and the new overall utility function is given as 


$$
\max U\left(\omega_{i}\right)=C \sum \omega_{i}-\sum f_{i}\left(\omega_{i}\right)
$$

where $C$ is a constant. In fact, the optimal solution of maximizing $U\left(P_{i}\right)$ is the same as the optimal solution of maximizing $f$. Each agent has a sub-utility function which is expressed as:

$$
\max U_{i}\left(\omega_{i}\right)=C \omega_{i}-f_{i}\left(\omega_{i}\right)
$$

Agents participate in the optimal process aiming to maximize their sub-utility function. Since the frequency response in each system depends on its parameters, a proper fitness function can be set for the agents, let $f_{i}\left(\omega_{i}\right)=$ $\frac{\partial U_{i}\left(\omega_{i}\right)}{\partial \omega_{i}}$, with $f_{i}$ as the fitness function and $\omega_{i}$ is the potential frequency measurement.

When the agents participate in the evolution game process, the fitness function of the $i_{t h}$ agent evolves with the average fitness $\bar{f}$. If $f_{i}\left(\omega_{i}\right)>\bar{f}$, the amount of individuals choosing the $i_{t h}$ agent as the habitat would be increased. If $f_{i}\left(\omega_{i}\right)<\bar{f}$, the amount of individuals choosing the $i_{t h}$ agent as the habitat would be decreased. With the changing of $\omega_{i}, f_{i}\left(\omega_{i}\right)$ and $\bar{f}$ would also be changed, which would affect $\omega_{i}$ conversely. The RD model evolves until it reaches the steady state, and the steady equilibrium point is the solution of overall utility function.

\section{Notations for SAA}

Some necessary notations in this paper when applying SAA are introduced as below. The number of power systems is $K$. The output frequency in $m^{t h}$ system is denoted as $\operatorname{freq}_{m}(t)$, which is a function of time. There are $k$ parameters, $y_{1}, y_{2}, \ldots, y_{n}$, to be tuned in $m^{\text {th }}$ system, which is expressed as vector $Y_{m}=\left[y_{1}, y_{2}, \ldots, y_{k}\right]^{T} . Y=\left\{Y_{1}, Y_{2}, \ldots, Y_{K}\right\}$ is the set of the solution of all the parameters to be tuned in $K$ systems. $Y^{i}=\left\{Y_{1}^{i}, Y_{2}^{i}, \ldots, Y_{K}^{i}\right\}$ is the set of the solution of all the parameters to be tuned in $K$ systems in $i^{\text {th }}$ iteration of Simulated Annealing Algorithm. $w_{1}, w_{2}, w_{3}$ are the weights in cost functions which can be tuned according to the requirements of designers. $y_{m l}$ is the $l^{\text {th }}$ parameters to be tuned in $m^{\text {th }}$ system, $y_{\text {mlmin }}$ and $y_{\text {mlmax }}$ are the lower bound and upper bound of $y_{m l}$ respectively. Cost function in SA algorithm is $Q$ and $\Delta Q$ is the change in Cost Function between $(i+1)^{\text {th }}$ and $i^{\text {th }}$ iteration. State Acceptance Function is denoted as $S$. Temperature Renew Function is denoted as $T_{k}$ and $T$ is parameter temperature in SA algorithm [49].

\subsection{Functions and Parameters in Simulated Annealing algorithm}

\subsubsection{Cost Function}

Cost function $Q$ is the core of the algorithm. The objective of the Simulated Annealing algorithm is to find the optimal solution that satisfies and minimizes the cost function in the system studied.

Normally, in control systems, the goal is to minimize the error between output and reference signal. However, in the power system studied, the goals are set as:

1. Reduce undershoot of the frequency response (nadir)

2. Reduce recovery time of the primary frequency control (settling time)

3. Minimize error between the frequency and $50 \mathrm{~Hz}$.

Thus, a cost function is required to fulfill the requirements in a power system.

Simulated Annealing Algorithm for the power system case is used to solve the following constrained optimization problem:

$$
\begin{aligned}
\min & Q(Y) \\
\text { subject to } & y_{\text {mlmin }} \leq y_{m l} \leq y_{\text {mlmax }}, m \in\{1,2, . ., K\}, l \in\{1,2, \ldots, k\}
\end{aligned}
$$

where $x_{m l}$ is the $l^{\text {th }}$ parameters to be tuned in $m^{\text {th }}$ system, $y_{\operatorname{mimin}}$ and $x_{\operatorname{mlmax}}$ are the lower bound and upper bound of $y_{m l}$ respectively.

$Q(Y)$ is cost function which is a function of set $Y$. More specifically, $Q(Y)$ for $K$ power systems is designed as below.

$$
Q(Y)=w_{1} \times E+w_{2} \times C+w_{3} \times U+w_{4} \times T+w_{5} \times S
$$


$50 \mathrm{~Hz}$

In $Q(Y), E=\sum_{m=1}^{N} \int_{0}^{\infty}\left(\operatorname{freq}_{m}(t)-50 H z\right)^{2} d t$ measures the sum of errors between $f r e q_{m}(t)$ and reference signal

$C=\sum_{m=1}^{K} Y_{m} \cdot Y_{m}^{T}$ measures the sum of square of control signal in all the power systems.

$U=\sum_{m=1}^{K} U_{m}$ is a measurement of undershoot in $\mathrm{K}$ power systems.

$T=\sum_{m=1}^{K} T_{m}$ is a measurement of settling time in $\mathrm{K}$ power systems.

$S=\sum_{m=1}^{K} \frac{1}{2}\left(1+\operatorname{sgn}\left(\right.\right.$ freq $\left.\left._{m}(t)-50 H z\right)\right) \times \int_{0}^{\infty}\left(\text { freq }_{m}(t)-50 H z\right)^{2} d t$ is the penalty item to avoid overshoot, which is not desired in output frequency.

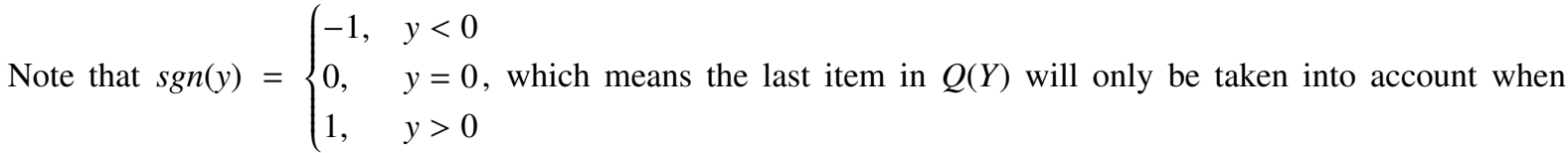
freq $_{m}(t)-50 \mathrm{~Hz}>0$. Besides, $w_{5} \gg w_{i}, i \in\{1,2, \ldots, 4\}$ will make $Q(Y)$ is much more larger when there is an overshoot in the output frequency. It will make the solution with overshoot less possible to be accepted since it will have a larger cost function due to the penalty item. The weights selected are $w_{1}=1, w_{2}=1000, w_{3}=100 w_{4}=1000$ $w_{5}=1000$ respectively.

Appendix A presents the fundamentals of the SAA, and Appendix B shows the pseudo algorithm used.

\section{Power System Preliminaries}

\subsection{Power System Frequency Response}

The frequency control in a power system after a large disturbance is performed in different stages and time frames, namely inertia frequency, Frequency Containment Reserve (FCR) (primary control) and Frequency Restoration Reserves (FRR) (secondary control).

Inertial response is inherent in the system due to rotating mass of machines synchronously connected providing counter response within seconds to oppose the frequency deviation following a loss of generation or a load event.

In a synchronous system, in the case of losing a generating unit, the frequency drops because of the imbalance between generation and load. The system frequency response is reflected in the power system instantaneously. During the first period, the inertial response of the spinning machines in the entire system reacts, releasing or storing kinetic energy tending to reduce the frequency deviation. System inertia is defined as the total amount of kinetic energy stored in all rotating masses.

The inertial constant of an individual generator can be interpreted as the time that generator can provide full output power from its stored kinetic energy; it can have values between 2 to 9 seconds.

Beyond the inertial response, the frequency is stabilized and then restored to the nominal frequency by the FCR (governor action) and secondary controllers, respectively. FCR acts as a proportional controller avoiding large frequency deviations; however a steady state error is still remained. The response of this control is given in seconds (typically $<30$ s).

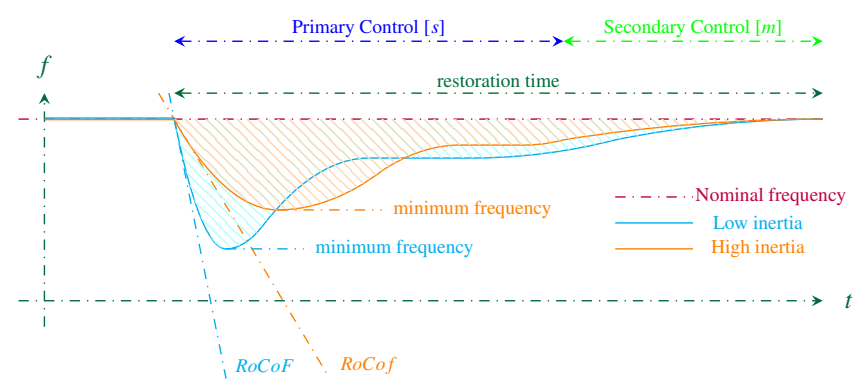

Figure 2: Frequency Response after Frequency Event

FRR returns the frequency back to its nominal value and also restores the reserves; its deployed time frame is given in minutes.

Figure 2 shows the dynamic response of the system frequency after disconnection of one generator. In the figure 
two cases are represented, one with low inertia and one with high inertia. Both differ in the Instantaneous Frequency Deviation (IFD) and Rate of Change of Frequency (RoCoF).

\subsection{System Model}

A power system can be conveniently described by a diagram consisting of a set of edges and vertices. An undirected graph $\mathcal{G}=(\mathcal{V}, \mathcal{E})$ is used to represent an electrical network modeled by $n_{b}$ buses, denoted by the set of network nodes by $\mathcal{V}=\left\{1, \cdots, n_{b}\right\}$, with a subset of $n_{g}$ machines, $\mathcal{E}$ is the set of power transmission lines, and the voltages at these buses $\bar{U}_{n_{g}}$. The dynamic of the $i: t h$ generator is given by:

$$
\dot{\omega}_{i}=\frac{1}{M_{i}}\left(P_{i}^{m}-P_{i}^{e}-D_{i} \omega_{i}\right)
$$

where $\omega_{i}$ (in rad/s) is the rotor speed of generator $i, P_{i}^{m}$ is the mechanical power (p.u.), and $P_{i}^{e}$ is the electric power (p.u.), and $D_{i}$ is the load-damping constant. Furthermore, $M_{i}$ is given by

$$
M_{i}=\frac{2 H_{i}}{\omega_{i}} \frac{S_{n g i}}{S_{\text {base }}}
$$

where $S_{n g i}$ and $S_{\text {base }}$ stand for the nominal power of each generator and the power base respectively.

The inertia constant $H_{i}$ of the generator is defined by

$$
H_{i}=\frac{1}{2} \frac{J_{i} \omega_{m s}^{2}}{S_{n i}}
$$

where, $J_{i}$ is the total moment of inertia, $S_{n i}$ is the rated power of generator, $\omega_{m s}$ is the mechanical frequency.

\subsection{Primary Frequency Response Modeling}

The objective of a turbine governing system installed in a generating unit is to produce a desired power which is partly determined by the set value for the produced power and partly by a contribution originating from the frequency control. In this context, the latter is of interest.

Figure (3) shows a schematic diagram of the system model which combines the generator and load, the hydroturbine and, the electro-mechanical prime governor.

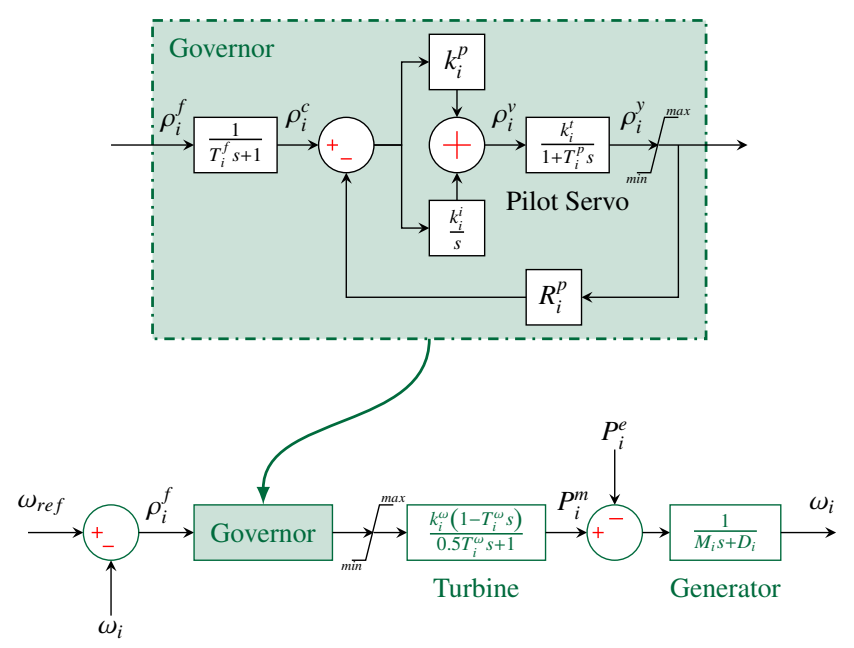

Figure 3: New Governor Model Connected to the Grid

The model including the governing system, the servo and the turbine depicted in Figure (3) is given by (10) and its state space model in (11): 


$$
\begin{aligned}
& \left\{\begin{aligned}
\dot{\omega}_{i} & =\frac{1}{M_{i}}\left(P_{i}^{m}-P_{i}^{e}-D_{i} \omega_{i}\right) \\
\dot{P}_{i}^{m} & =-2 k_{i}^{\omega} \dot{\rho}_{i}^{y}+\frac{2 k_{i}^{\omega}}{T_{i}^{\omega}} \rho_{i}^{y}-\frac{2}{T_{i}^{\omega}} P_{i}^{m} \\
\dot{\rho}_{i}^{y} & =\frac{1}{T_{i}^{p}}\left(k_{i}^{t} \rho_{i}^{v}-\rho_{i}^{y}\right) \\
\dot{\rho}_{i}^{v} & =k_{i}^{i} \rho_{i}^{c}-k_{i}^{i} R_{i}^{p} \rho_{i}^{y}+k_{i}^{p} \dot{\rho}_{i}^{c}-k_{i}^{p} R_{i}^{p} \dot{\rho}_{i}^{y} \\
\dot{\rho}_{i}^{c} & =\frac{1}{T_{i}^{f}}\left(\omega_{r e f}-R_{i}^{f} \omega_{i}-\rho_{i}^{c}\right)
\end{aligned}\right.
\end{aligned}
$$

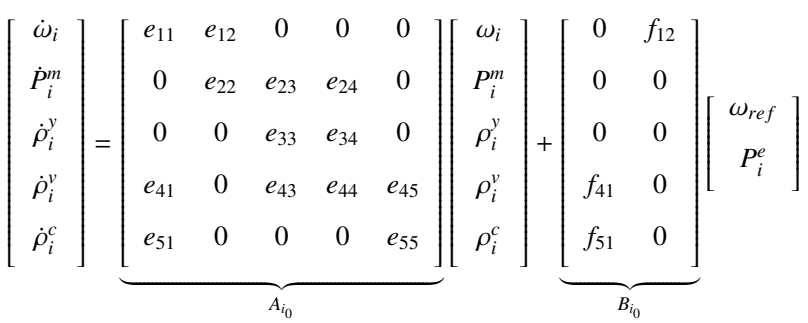

The respective terms utilized in (11) are summarized in the Table 1.

\begin{tabular}{|c|c||c|c|}
\hline Parameter & Value & Parameter & Value \\
\hline \hline$e_{11}$ & $-D_{i} / M_{i}$ & $e_{12}$ & $1 / M_{i}$ \\
$e_{22}$ & $-2 / T_{i}^{\omega}$ & $e_{23}$ & $2 k_{i}^{\omega} / T_{i}^{p}+2 k_{i}^{\omega} / T_{i}^{\omega}$ \\
$e_{33}$ & $-1 / T_{i}^{p}$ & $e_{24}$ & $2 k_{i}^{t} k_{i}^{\omega} / T_{i}^{p}$ \\
$e_{34}$ & $k_{i}^{t} / T_{i}^{p}$ & $e_{43}$ & $-k_{i}^{i} R_{i}^{p}+k_{i}^{p} R_{i}^{p} / T_{i}^{p}$ \\
$e_{41}$ & $-k_{i}^{p} R_{i}^{f} / T_{i}^{f}$ & $e_{44}$ & $-k_{i}^{p} R_{i}^{p} k_{i}^{t} / T_{i}^{p}$ \\
$e_{51}$ & $-R_{i}^{f} / T_{i}^{f}$ & $e_{45}$ & $k_{i}^{i}-k_{i}^{p} / T_{i}^{f}$ \\
$e_{55}$ & $-1 / T_{i}^{f}$ & $f_{12}$ & $-1 / M_{i}$ \\
$f_{41}$ & $k_{i}^{p} / T_{i}^{f}$ & $f_{51}$ & $1 / T_{i}^{f}$ \\
\hline \hline
\end{tabular}

Table 1: Terms of the Power system defined in (11)

where the constants $T_{i}^{p}, T_{i}^{\omega}, k_{i}^{i}, k_{i}^{p}, T_{i}^{f}, R_{i}^{p}, k_{i}^{t}$ stand for the servo pilot time constant, the water time constant, the integral controller constant, the proportional controller constant, the reset time constant, permanent droop and the servo pilot gain respectively.

\subsection{Stability Analysis}

Theorem: The power system (11) is stable for $k_{i}^{p} \in\left(0, \mu_{i}^{p}\right), k_{i}^{i} \in\left(0, \mu_{i}^{i}\right)$.

Remark: $\mu_{i}^{p}$ y $\mu_{i}^{i}$ are defined by the Routh-Hurwitz stability criterion through the coefficients of the characteristic polynomial.

Proof: The stability of (11) is determined by the eigenvalues of $A_{i_{0}}$. The roots of the characteristic polynomial of $A_{i_{0}}$ is given by (12).

$$
p_{1}(s)=\operatorname{det}\left[s \mathbf{I}-A_{i}\right]=\sum_{j=0}^{5} a_{j} s^{j}=0
$$

where $A$ is the state system matrix.

The characteristic polynomial (12) must be evaluated by the Routh-Hurwitz stability criterion. The coefficients of Routh-Hurwitz stability criterion are summarized in the Table 2. For a better understanding of the method see reference [50].

If these coefficients are strictly greater than zero, the system is said to be stable. The stable zone can be seen in Figure 4. Full restrictions problem can be seen in Appendix C. 
Table 2: Coefficients of the Routh-Hurwitz Criterion of the Power system defined in (11)

\begin{tabular}{|c|c||c|c|}
\hline Coefficient $(\cdot)$ & Shape & Coefficient $(\cdot)$ & Shape \\
\hline \hline$a_{5}$ & $p_{5}$ & $b_{1}\left(k_{i}^{p}, k_{i}^{i}\right)$ & $a_{3}-a_{2} a_{5} / a_{4}$ \\
$a_{4}\left(k_{i}^{p}\right)$ & $m_{4} k_{i}^{p}+p_{4}$ & $b_{0}\left(k_{i}^{p}, k_{i}^{i}\right)$ & $a_{1}-a_{0} a_{5} / a_{4}$ \\
$a_{3}\left(k_{i}^{p}, k_{i}^{i}\right)$ & $m_{3} k_{i}^{p}+n_{3} k_{i}^{i}+p_{3}$ & $c_{1}\left(k_{i}^{p}, k_{i}^{i}\right)$ & $a_{2}-b_{0} a_{4} / a_{1}$ \\
$a_{2}\left(k_{i}^{p}, k_{i}^{i}\right)$ & $m_{2} k_{i}^{p}+n_{2} k_{i}^{i}+p_{2}$ & $c_{0}\left(k_{i}^{i}\right)$ & $n_{0} k_{i}^{i}$ \\
$a_{1}\left(k_{i}^{p}, k_{i}^{i}\right)$ & $m_{1} k_{i}^{p}+n_{1} k_{i}^{i}+p_{1}$ & $d_{0}\left(k_{i}^{p}, k_{i}^{i}\right)$ & $b_{0}-c_{0} b_{1} / c_{1}$ \\
$a_{0}\left(k_{i}^{i}\right)$ & $n_{0} k_{i}^{i}$ & $e_{0}\left(k_{i}^{i}\right)$ & $n_{0} k_{i}^{i}$ \\
\hline \hline
\end{tabular}

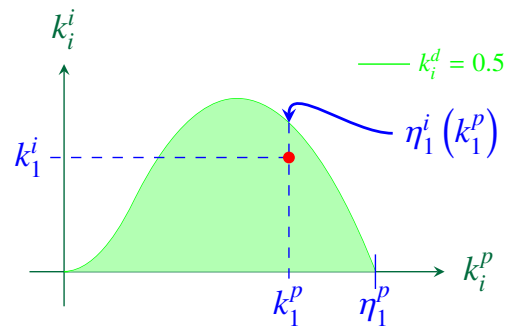

Figure 4: Stable Zone under Local PI Controller

\section{Hierarchical Management of Governors}

In this section, the main contribution of this paper is presented i.e. a hierarchical dynamic management strategy to improve the frequency response in power systems through a supplementary control in the hydro governors. This approach considers a network control system which provides the distributed control signals $x_{i}$ in each hydro governor involved. Moreover, there is a connecting graph of the control network.

\subsection{Supplementary Governor Signal Approach}

Figure 5 shows the addition of a supplementary signal added to the governor. The model is given by (13).

$$
\left\{\begin{aligned}
\dot{\delta}_{i} & =\omega_{i} \\
\dot{\omega}_{i} & =\frac{1}{M_{i}}\left(P_{i}^{m}-k_{i j}\left(\delta_{i}-\delta_{j}\right)-D_{i} \omega_{i}\right) \\
\dot{P}_{i}^{m} & =-2 k_{i}^{\omega} \dot{\rho}_{i}^{y}+\frac{2 k_{i}^{\omega}}{T_{i}^{\omega}} \rho_{i}^{y}-\frac{2}{T_{i}^{\omega}} P_{i}^{m} \\
\dot{\rho}_{i}^{y} & =\frac{1}{T_{i}^{p}}\left(k_{i}^{t} \rho_{i}^{y}-\rho_{i}^{y}-k_{i}^{t} x_{i}\right) \\
\dot{\rho}_{i}^{v} & =k_{i}^{i} \rho_{i}^{c}-k_{i}^{i} R_{i}^{p} \rho_{i}^{y}+k_{i}^{p} \dot{\rho}_{i}^{c}-k_{i}^{p} R_{i}^{p} \dot{\rho}_{i}^{y} \\
\dot{\rho}_{i}^{c} & =\frac{1}{T_{i}^{f}}\left(\omega_{r e f}-R_{i}^{f} \omega_{i}-\rho_{i}^{c}\right)
\end{aligned}\right.
$$

\subsection{Control Architecture}

The proposed new supplementary control with the dynamic adjustment of the hydro governor is shown in Figure 6 for $n$ interconnected areas/machines. The main concept behind the proposed structure is based on sharing the frequency deviation measured from the $\mathrm{CoI}$ (Center of Inertia) reference frame of all the available hydro governors in the system. It is assumed that all the local controllers have already a designed local PI controller (hydro governors) in charge of reducing the frequency deviation.

The addition of supplementary control action based on the RoCoF measurement in the governors provides an output that is proportional to it. For a step change in the frequency, during the initial stage of the event, the RoCoF result would have a larger value, and thus it is effective for arresting the frequency response. However, since the RoCoF decreases in time due to the governor's participation, its contribution becomes less. 


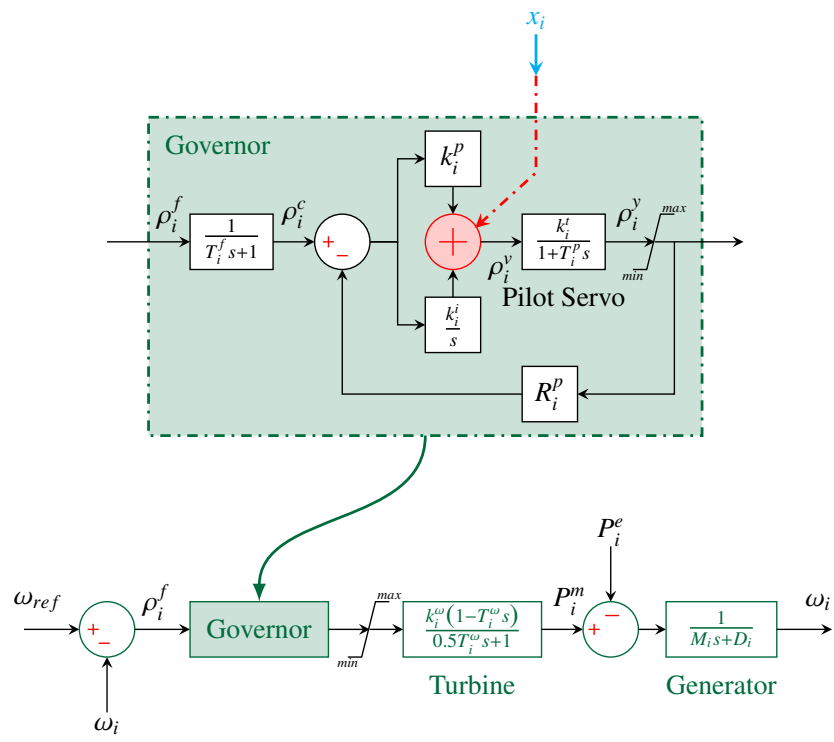

Figure 5: New Governor Model Connected to the Grid with Supplementary input $x_{i}$

The distributed control action introduced by the RD is given to the hydro governors components according to the individual capacities and parameters. The governor transmits its action through the servo - turbine combination and the inverse of the droop gain as well.

The aim is to show that the proposed approach, as an distributed intelligent agent cooperating with other hydro governors, thus creating a distributed intelligent system, can improve the frequency system response.

Typical Automatic Generation Control (AGC) aims to eliminate the frequency deviation and keep the tie-line deviation under the power operation limits. In contrast to the presented application, the supplementary control based on the distributive RoCoF measurement, aims to only improve the primary control and distribute and optimize the response of each governor and the total frequency response as a cooperative game. Note that a steady state error remains in the system since the AGC is not activated.

\subsection{Measurement metrics}

The CoI is used to have an estimated measurement of the frequency of an entire interconnected system. The CoI is computed based on the individual speeds $\omega_{i}$ and the inertia constants of the synchronous generators $H_{i}$.

Assuming the set $\mathcal{G}$ of synchronous generators, the expression to compute the $\mathrm{CoI}$ is:

$$
\omega_{C o I}=\frac{\Sigma_{i \in \mathcal{G}} H_{i} \omega_{i}}{\Sigma_{i \in \mathcal{G}} H_{i}}
$$

In the same way, the RoCoF measurement in the $\mathrm{CoI}$ reference is defined as

$$
\frac{d \omega_{C o I}}{d t}=\frac{\Sigma_{i \in \mathcal{G}} H_{i} \frac{d \omega_{i}}{d t}}{\Sigma_{i \in \mathcal{G}} H_{i}}
$$

The RoCoF has been calculated as the derivative of the frequency with a sampling period enough to in order to provide the measurement, the aggregation and the power injection response within $500 \mathrm{~ms}$ as recommended the EIRGRID grid code.

\subsection{Performance metrics}

Following a disturbance in the system, in particular given a negative step disturbance such as a sudden load increase or generation drop at $t=t_{1}$, the following metrics are defined for quantifying the action of the distributed control action: 


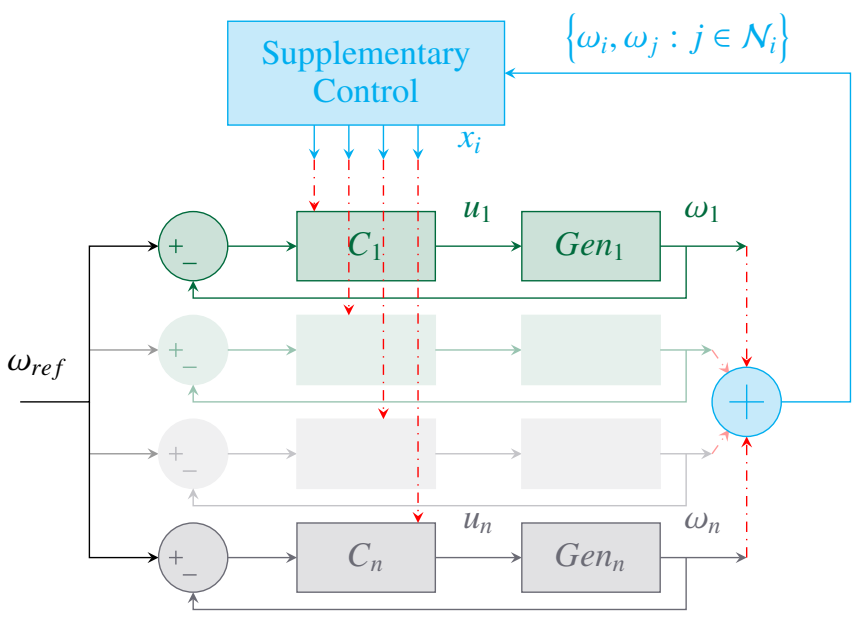

Figure 6: Supplementary Control Architecture

- Nadir is the maximum dynamic frequency deviation following an active power disturbance/contingency. It is dominated by the system inertia and governors response. Obtaining the optimal governor parameters the frequency nadir can be reduced.

- Nadir time is the associated time $t=t_{2}$ to the nadir occurrence.

- Settling time $t=t_{3}$ is used to study the transient condition and to having a time-mark to evaluate the control action on the settling frequency.

Figure 7 depicts these metrics in the frequency response. The objective is to reduce the nadir and minimize the time difference between $t_{2}$ and $t_{3}$ to an appropriate margin where it is improving the response reaction and avoiding any oscillations in the response.

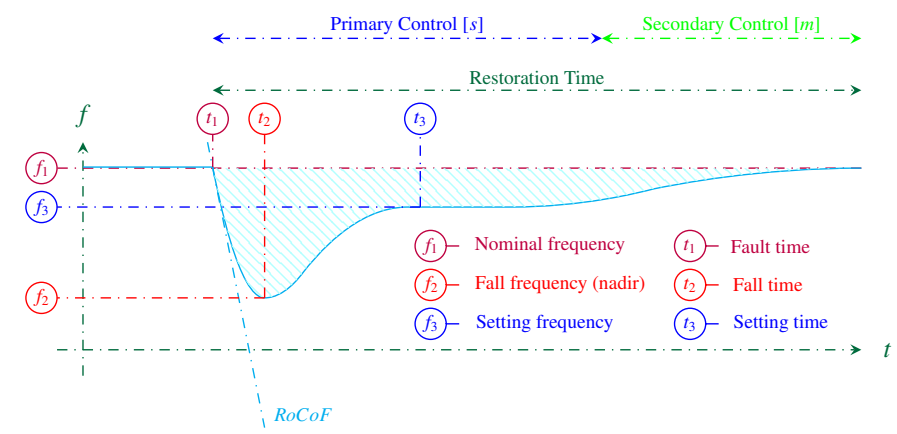

Figure 7: Power System Frequency Response

\subsection{Replicator Dynamics}

The population of most successful players (compared with the average) tend to grow, while the lease ones decline. The success of players who have chosen the $i^{\text {th }}$ strategy is determined by a function $f_{i}$, which is a fitness function in behavioral ecology.

$$
\dot{x}_{i}=\beta x_{i}\left(\omega_{i}-\bar{\omega}\right)
$$

where $x_{i}$ is the population playing the $i^{\text {th }}$ strategy, $\bar{f}=\left(\frac{1}{P}\right) \sum_{i=1}^{N} x_{i} f_{i}$ is the average fitness, $P$ is the total population $\left(P=\sum_{i=1}^{N} x_{i}\right)$, and $\beta>0$ is a parameter related to the population growth rate.

Moreover, the fitness function is defined as a function of the difference between the $\omega_{i}$ of the $i^{\text {th }}$ generator and its corresponding reference as follows: 
$\max u(x): \Sigma_{i \in \mathcal{G}}\left(a_{i} \omega_{i}+b_{i} \dot{\omega}_{i}+c\right) x_{i}, \quad\left\{a_{i}, b_{i}\right\} \in \mathbb{R}_{<0}$

subject to $g_{1}(x): \Sigma_{i \in \mathcal{G}} x_{i}=1$

where $a_{i}$ and $b_{i}$ are the $i^{\text {th }}$ parameters to be tuned in $i^{\text {th }}$ power system. $\dot{\omega}_{i}$ owned information about RoCoF, and $c>0$. $u(\cdot)$ is cost function and strictly concave, and $g_{1}(\cdot)$ is the constraint related with proportional action in each power system.

where $\dot{d}_{\omega_{i}}$ is the derivative term and provides information about RoCoF. Therefore, derivative action is allows the controller to avoid or reduce overshoots in the fall time.

\subsection{Equilibrium Points}

The power system model consisting of $N$ governor-generators and controlled via replicator dynamics is described by (10). The equilibrium point of this system is denoted by $\left(\omega^{*}, P^{m *}, \rho^{y *}, \rho^{v *}, \rho^{c *}\right)$ where a desirable equilibrium point is achieved when each governor-generator reaches the settling frequency.

The complete characterization of the equilibrium point is given by:

$$
\left[\begin{array}{c}
\omega_{i}^{*} \\
P_{i}^{m *} \\
\rho_{i}^{y^{*}} \\
\rho_{i}^{v^{*}} \\
\rho_{i}^{c *}
\end{array}\right]=\left[\begin{array}{c}
\frac{k_{i}^{\omega} \omega_{r e f}^{p}}{R_{i}^{p} D_{i}}-\frac{P_{i}^{e}}{D_{i}} \\
\frac{k_{i}^{\omega} \omega_{r e f}}{R_{i}^{p}} \\
\frac{\omega_{r e f}}{R_{i}^{p}} \\
\frac{\omega_{r e f}}{R_{i}^{p} k_{i}^{t}} \\
\omega_{r e f}
\end{array}\right]
$$

\subsection{Stability Analysis}

The passivity theory is used for the interconnected system analysis [51]. In this case, Figure 8 shows feedback interconnected between Power System $\Sigma^{p}$ and Centralized Control via RD $\Sigma^{c}$.

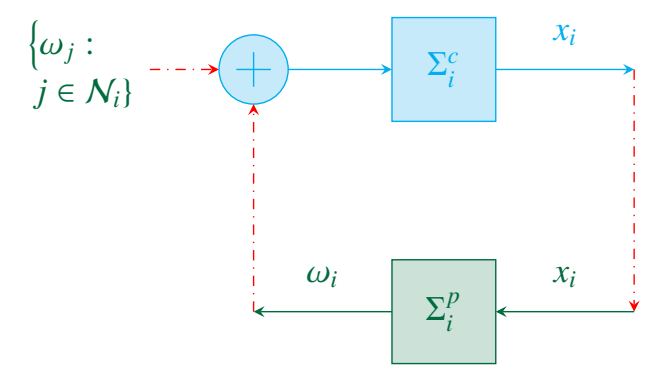

Figure 8: Interconnected Systems $\Sigma_{i}^{c}$ and $\Sigma_{i}^{p}$

The equilibrium point at the origin of the feedback interconnected system is made stable by using the following candidate function:

$$
V_{2}\left(d_{\omega}\right)=-\frac{1}{\beta} \sum_{i=1}^{N} \omega_{C o I} \ln \left(\frac{d_{\omega_{i}}^{*}+\omega_{C o I}}{\omega_{C o I}}\right)
$$

The derivative of $V_{2}\left(d_{\omega}\right)$ along the trajectories of $\Sigma_{2}$ is given by $\dot{V}_{2}\left(d_{\omega}\right)$.

The proposed function guarantees that following the hierarchical controller RD, the system remains in a stable region (more restrictively, asymptotically stable since $V_{2}\left(d_{\omega}\right)$ is decreasing with time). Moreover, having a stable region for both the local controller and the RD hierarchical control, the system will remain stable. Appendix D presents the proof of the stability function. 


\section{Study Cases}

\subsection{Three Mass Areas}

In order to test the methodology used, a test system has been created following the parameters in reference [11]. It represents a Nordic equivalent for frequency studies conformed by three areas, as depicted in Figure 9.

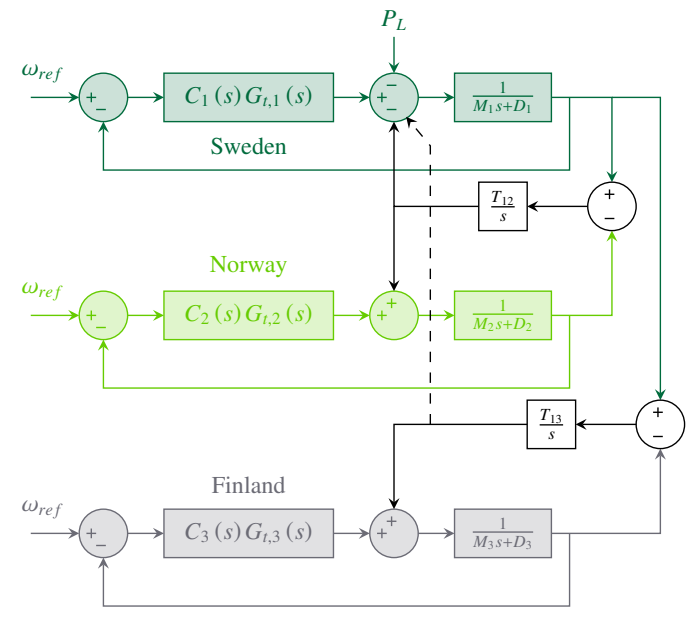

Figure 9: Three Mass Areas: block diagram

The parameters of each area, including the default governor settings, and the power production in the system are shown in Tables 3 and 4 respectively. Additionally, it is shown the 1-area aggregated model represents the entire frequency model system. Note that the default parameters do not contain the derivative controller constant.

Table 3: Hydro Governor Parameters

\begin{tabular}{|c|c|c|c|c|}
\hline Parameter & Aggregated Model & Sweden & Finland & Norway \\
\hline \hline$k_{i}^{p}$ & 1.6 & 0.25 & 0.08 & 1.27 \\
\hline$k_{i}^{i}$ & 0.175 & 0.0417 & 0.0133 & 0.141 \\
\hline$R_{i}^{p}$ & 0.133 & 0.236 & 1.25 & 0.236 \\
\hline$T_{i}^{p}$ & 0.2 & 0.2 & 0.2 & 0.2 \\
\hline$T_{i}^{\omega}$ & 1.01 & 1.4 & 1.4 & 0.7 \\
\hline$M_{i}$ & 9.68 & 4.65 & 1.93 & 3.25 \\
\hline$D_{i}$ & 0.517 & 0.246 & 0.087 & 0.184 \\
\hline
\end{tabular}

Table 4: Power Production Per Country

\begin{tabular}{|c|c|c|c|}
\hline Production & Sweden & Norway & Finland \\
\hline \hline$M W_{i}$ & 11620 & 17825 & 2028 \\
\hline$W_{i}^{k i n}$ & 112605 & 81177 & 48187 \\
\hline
\end{tabular}

where $W^{k i n}$ is kinetic energy of the system.

The obtained controllers parameters from the SAA are given in Table 5. 
Table 5: Obtained Controller Parameters by SAA

\begin{tabular}{|c|c|c|c|}
\hline Plant & $k_{i}^{p}$ & $k_{i}^{i}$ & $k_{i}^{d}$ \\
\hline \hline Sweden & 0.56 & 0.07 & 1.85 \\
\hline Norway & 0.80 & 0.05 & 2.34 \\
\hline Finland & 0.07 & 0.02 & 1.05 \\
\hline
\end{tabular}

\subsubsection{Determination of Local Stability Area of the Default System}

By solving the conditions expressed in Section (4.4), a second-degree (order) equation corresponding to a hyperbole in the $k p-k i$ plane is identified, indicating the locations of the maximum integral and proportional gains of each system respectively, or in other words, a stable region for the parameters $k p-k i$ is delimited. Figure 10 shows the evaluation of the stability conditions for the three areas and the aggregated model in the Nordic test system. Since each region has different parameters, the stability regions also differ. However, the full aggregated system, which is the representation of whole system, shows the merging in one stability region. Figure 10, also includes an unstable point to show the stability boundaries.

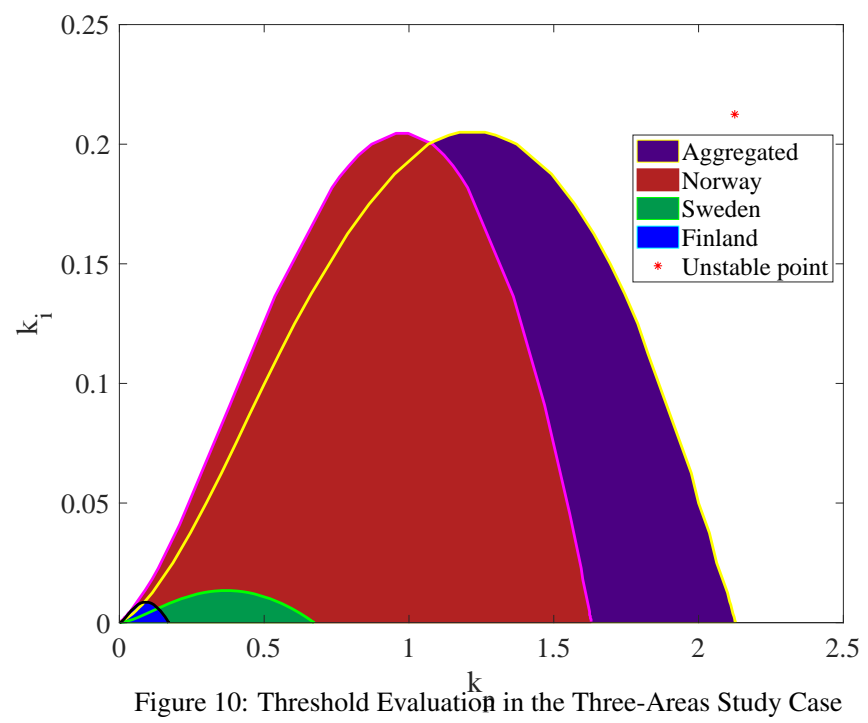

Additionally, the Nyquist plots are included to confirm the stability. Figure 11a shows the trajectories of all the regions including the aggregated system and the unstable case. Moreover, Figure 11b shows the zoom in of the Nyquist trajectories. Since none of the cases encloses the $(-1,0)$ point, they can be considered stable, contrary to the unstable case which encloses the $(-1,0)$ point. 


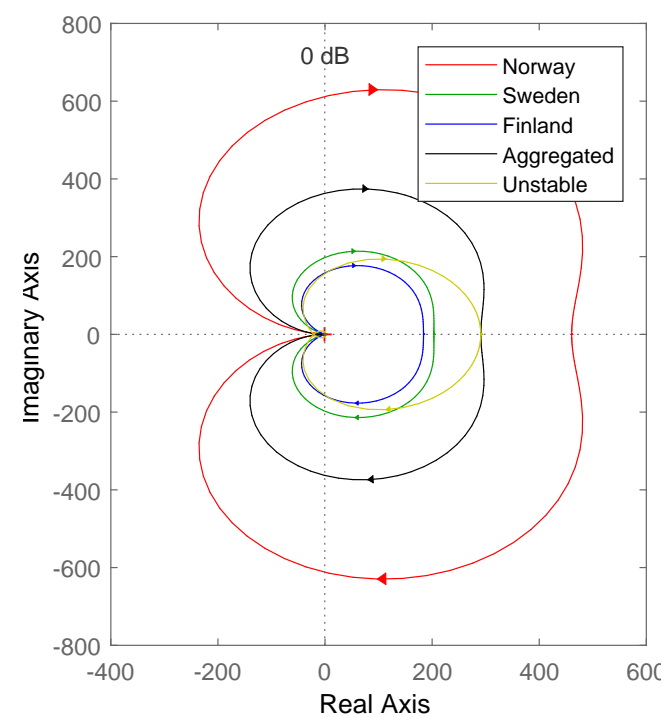

(a) Nyquist Diagram

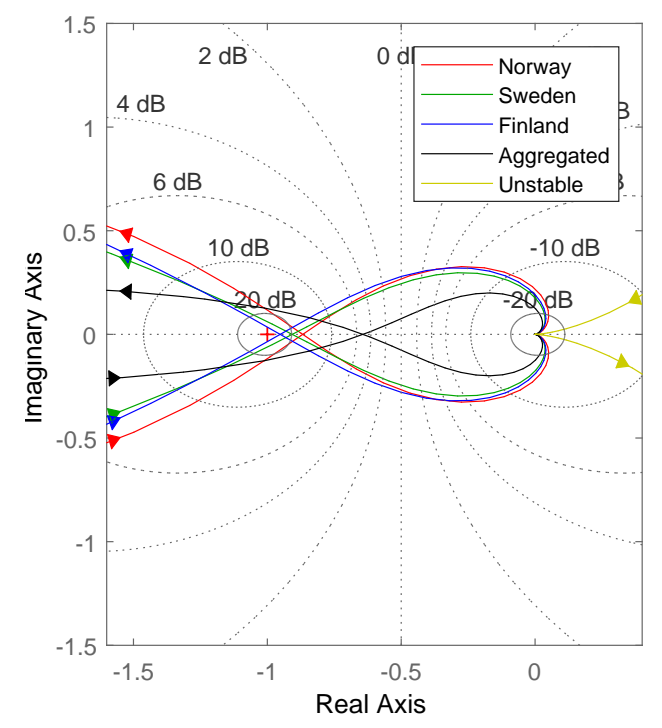

(b) Nyquist Diagram: Zoom

Figure 11: Nyquist Stability Plots

\subsubsection{Frequency Response}

By applying the SAA, the optimal governor parameters have been found based on the criteria established in (6). Figure $12 \mathrm{a}, 12 \mathrm{~b}$, and $12 \mathrm{c}$ show the time response comparison between the parameters obtained by the SAA and the default parameters in each area of the system. As can be seen in Figures 12a to 12c the application of the SAA, as a parameters optimizer, improves the frequency response drastically, reducing the overshoot and settling time. Additionally, in Figure 12d, the CoI time response is given. 


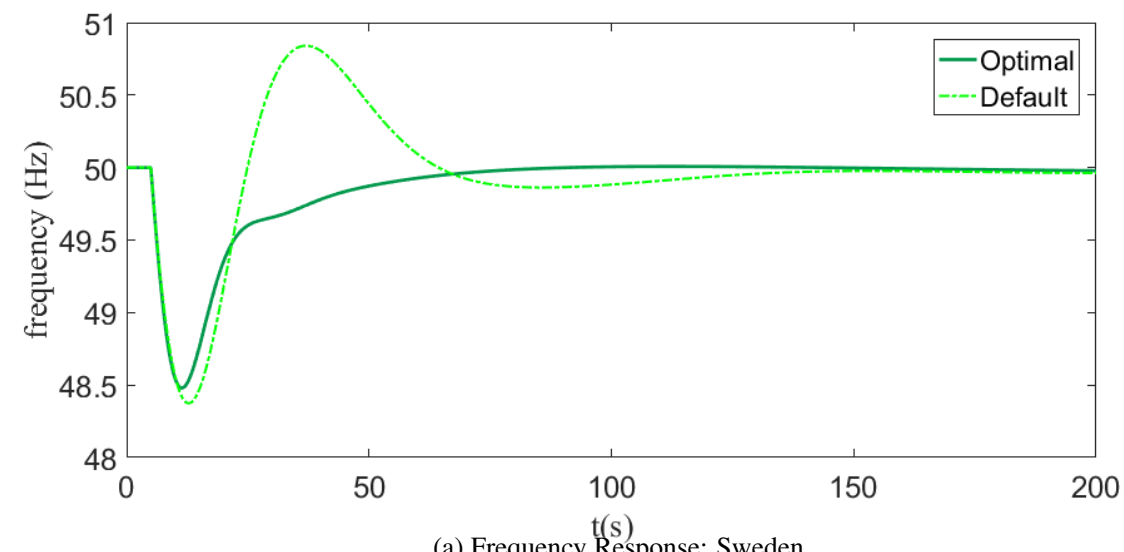

(a) Freauencv Response: Sweden

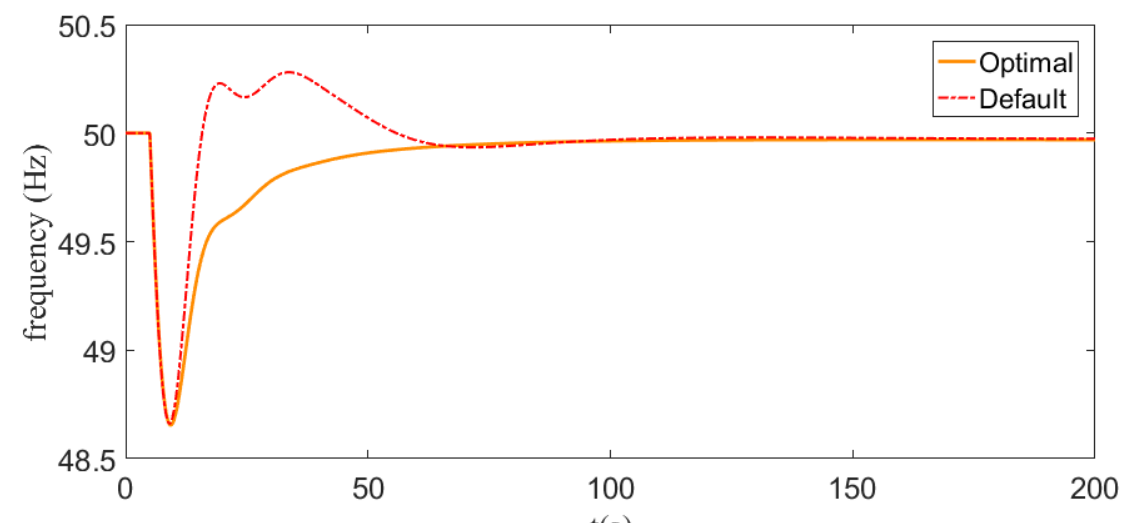

(b) Frequency Response: Norwav

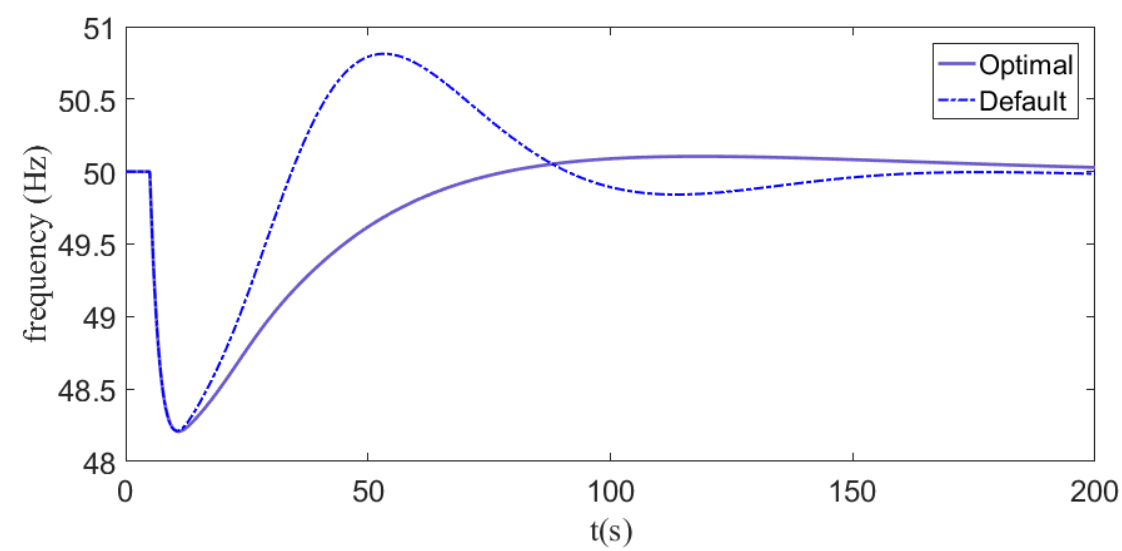

(c) Frequency Response: Finland

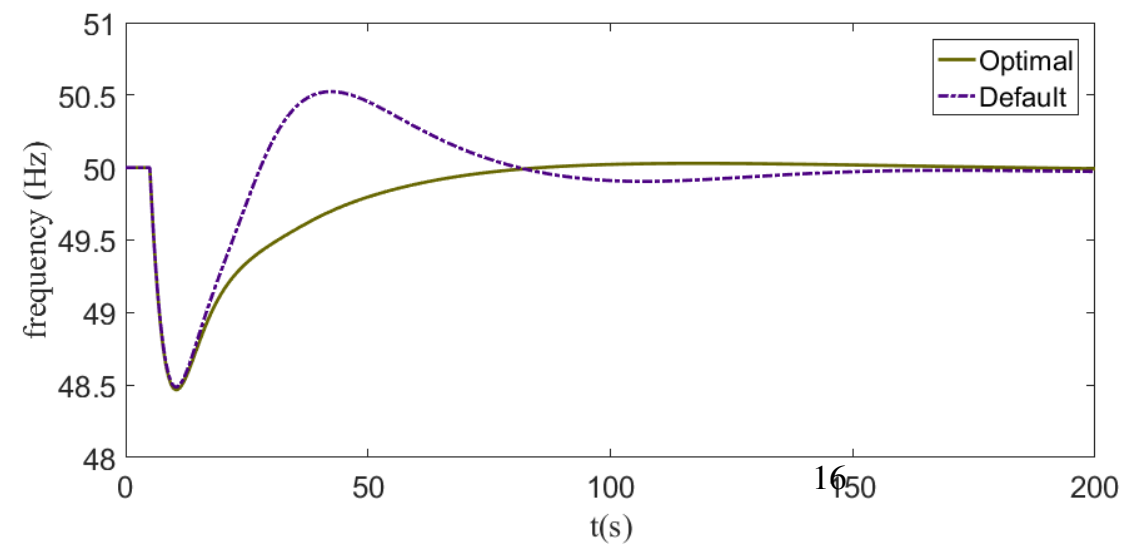

(d) Frequency Response: $\mathrm{CoI}$

Figure 12: Frequency Response: Three Areas System 
Table 6 shows the performance metrics of both the default (def) and optimized systems. The optimal SAA application has clearly improved the response of the default system. The relative settling time and overshoot have been reduced by $7 \%$ and $30 \%$ respectively in the overall system response.

Table 6: Default Parameters vs Optimal based on SAA

\begin{tabular}{|c|c|c|c|c|}
\hline Parameter & CoI & Sweden & Finland & Norway \\
\hline \hline$t_{s}-d e f$ & 139.91 & 117.95 & 146.80 & 83.92 \\
\hline$t_{s}-s a a$ & 132.49 & 65.22 & 165.34 & 68.06 \\
\hline$o_{v}-d e f$ & 1.1 & 1.75 & 1.65 & 0.6 \\
\hline$o_{v}-s a a$ & 0.07 & 0.05 & 0.15 & 0.06 \\
\hline nadir - def & 48.48 & 48.49 & 48.5 & 48.51 \\
\hline nadir - saa & 48.46 & 48.47 & 48.48 & 48.58 \\
\hline nadir $_{t}-$ def & 11.38 & 9.51 & 10.93 & 9.35 \\
\hline nadir $_{t}-$ saa & 11.13 & 9.48 & 11.01 & 9.41 \\
\hline
\end{tabular}

However, due to the absence of the derivative part in the system, it is necessary to include the derivative term in the optimization process for comparing it to the RD method. By including the derivative part and optimizing the values through the SAA method, the optimal response (in blue) in Figure 13 is obtained. Having obtained the optimal $k_{p}$ and $k_{i}$ parameters, it is now included the dynamic derivative part by RD response is given in green in Figure 13 as well. Both time responses are under the CoI frame. Additionally the RD response in each area is shown in Figure 14.

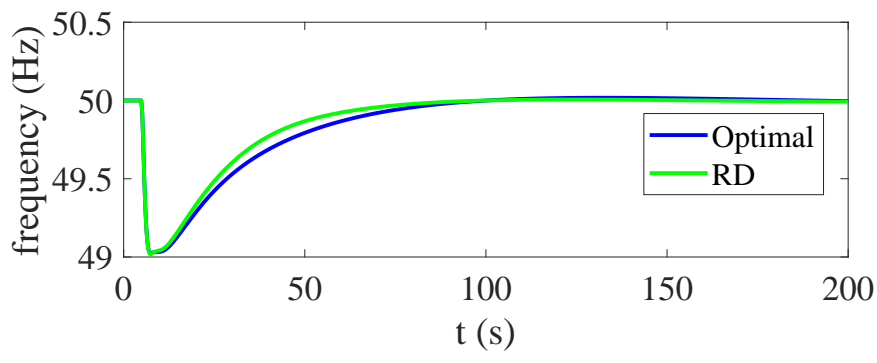

Figure 13: Frequency Response: Replicator Dynamics vs Optimal SSA

Table 7 shows the comparison between the SAA optimal parameters and the application of RD following the RoCoF measurements. From Figure 13, several observation can be made: (i). having optimized all the governing parameters $\left(k_{p}, k_{i}\right.$ and $\left.k_{d}\right)$ it is possible to obtain an adequate response and a clear improvement of the system frequency response, however the optimization process is based on an off-line technique, which has the drawback that by a change in the system parameters, it requires a new optimization procedure. (ii) The aim of this paper is to improve the time reaction of the governor response, therefore the addition of a derivative control part by the two methods. Recalling that the derivative part is quite sensitive and the tuning requires a rigorous process. (iii) Additional rate of change (derivative) of the error action improves settling time and stability of the system. This slows the rate of change of the controller output and therefore reduces the magnitude of the overshoot (produce by the integral action) and rise time. The wrong or excessive derivative action will cause an oscillatory approach that can persist after reaching a new operating point. (iv). The RD application, which is based on the RoCoF measurement, improves the time reaction of the governors as well, and it can be applied without any post-processing time if the system change. Note, a communication system is assumed to coordinate the actions of the various controllers. (v). Both ways of control suppressed the undesirable oscillations that might be caused by the over-tuning of the controllers. 
Table 7: Optimal Parameters vs RD

\begin{tabular}{|c|c|}
\hline Parameter & CoI \\
\hline \hline$t_{s}-s a a$ & 110.08 \\
\hline$t_{s}-r d$ & 77.12 \\
\hline$o_{v}-s a a$ & 0.04 \\
\hline$o_{v}-r d$ & 0.02 \\
\hline nadir $-s a a$ & 49.02 \\
\hline nadir $^{-r d}$ & 49.02 \\
\hline nadi $_{t}-s a a$ & 7.85 \\
\hline nadi $_{t}-r d$ & 7.62 \\
\hline
\end{tabular}

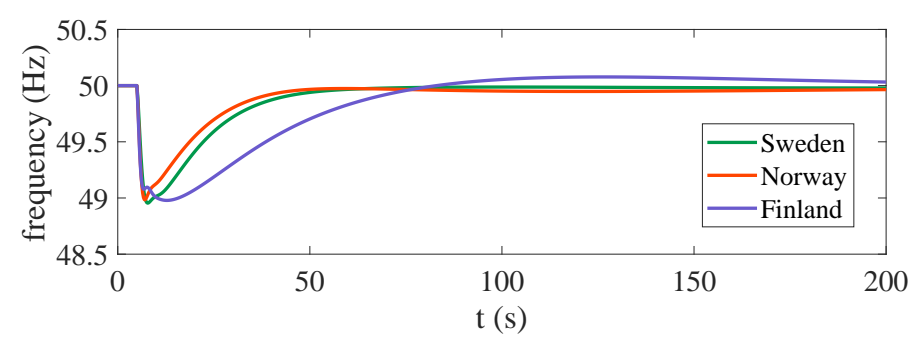

Figure 14: Frequency Response: Replicator Dynamics in Each Area

Figure 15 shows the evolution of the distributed control actions (wealth) under the scenario described above using a complete graph, i.e., individuals have full information. In this case, the evolution of the distributed control is described initially for an increasing action according to the RoCoF variation. Since each area has different parameters, (e.g. inertia constant, turbine parameters) the distributed action is also different and as expected. For instance, in the case of the action which is applied to Finland $x_{3}$ (in purple), the action is smaller than the other control signals that are distributed to Sweden $x_{1}$ (in green) and Norway $x_{2}$ (in orange). It is also observable that the control effort reacts as expected having the optimal dynamic adjustment and avoiding any undesirable oscillation and respecting the turbine saturation limits. The control action is diminished insofar the frequency gets re-established.

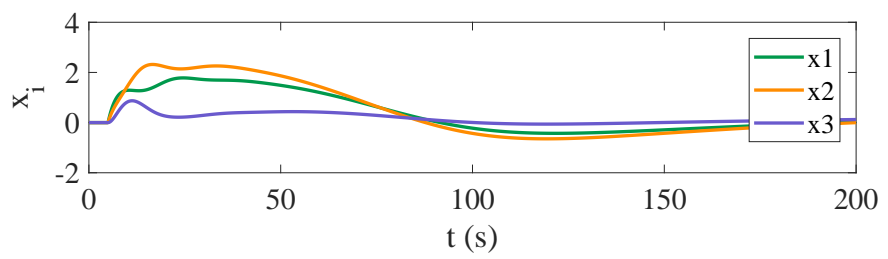

Figure 15: Wealth Evolution of Each Individual under RD

\subsubsection{Replicator Potential Function}

The potential function and its projection over the simplex are presented in Figure 16. Maximum point is reached based on the maximization function subject to the constraints given by the proposed simplex formulation. The RD usage and convergence to Nash equilibria have been proved in [52]. 


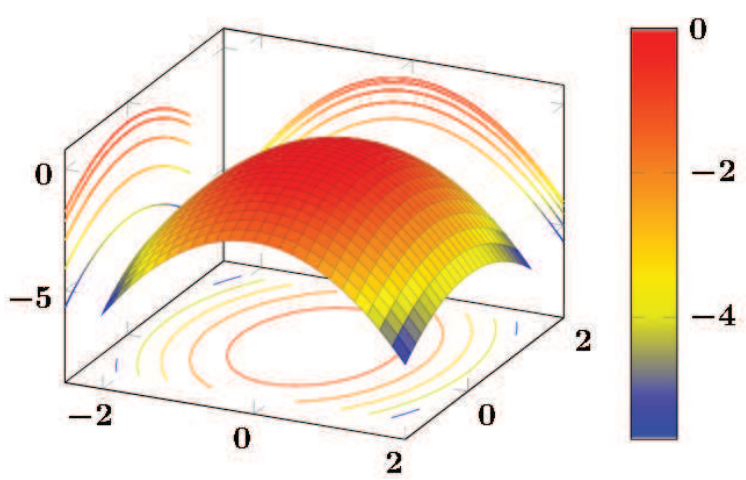

Figure 16: Threshold Evaluation in the Three-Areas Study Case

\subsubsection{Communication Delay Impact}

Since the RD application requires a communication channel layer, it is necessary to study the impact of the delay $\tau_{s}$ in the communication. Figure 17 shows the impact of the delay on the distributed variable $k_{d}^{i}$ in the system. Even though each sub-system has a similar value, the sub-system which is more affected by the delay is Finland since its limits of stability are lower. Additionally, Figure 17 points out the separation of the stability regions. For instance the maximum delay tolerated in Norway, Sweden and Finland is $1.23 \mathrm{~s}, 1.19$ and 1.18 respectively. After those values the system are in a unstable region and the control action is not effective anymore. Note that the red point denotes the separation and not the optimal value of $k_{d}^{i}$. Such maximum delays have been calculated applying Neutral Delay Differential Equations (NDDE), but the proof is out of the scope of this document. It can be seen that a delay of 600 $\mathrm{ms}$, can cause an oscillatory behavior, but the unstable region has not been reached.

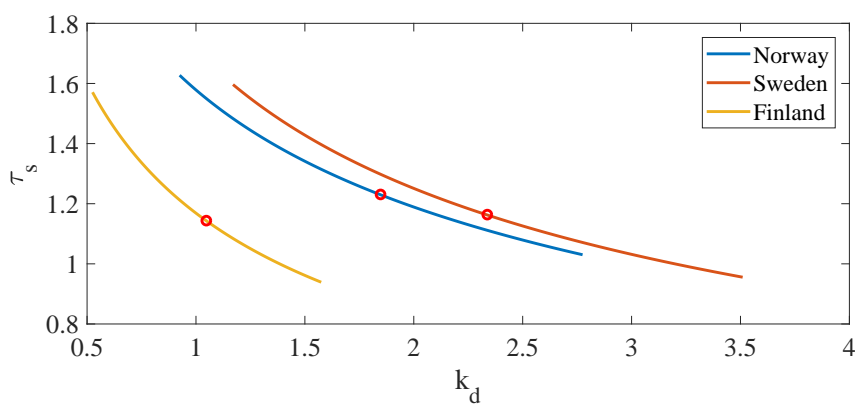

Figure 17: Delay instability boundaries per system area

The frequency response in the CoI reference with two delays in the communication channel is shown in Figure 18. The delay in the communication channel cannot only invoke undesirable oscillations but cause a different reaction in the hydro governor reaching nadir values that can alter the protection systems. 


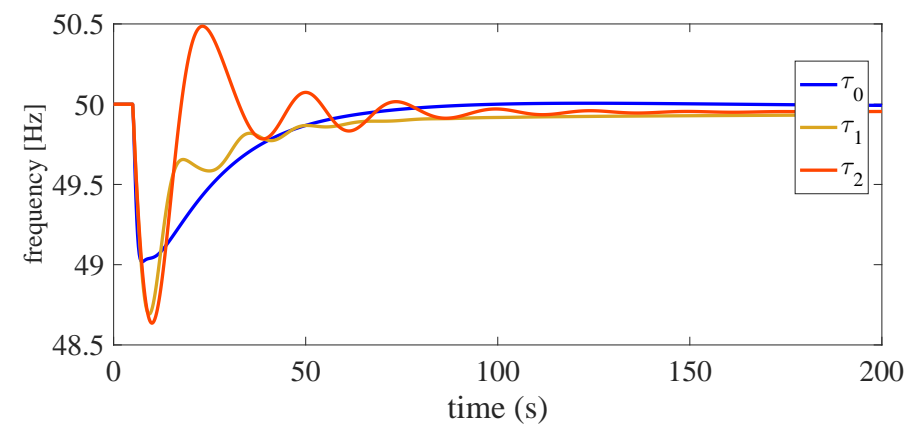

Figure 18: CoI Frequency Response: Increasing delay

\subsubsection{Inertia Variation Comparison}

In order to show the robustness of the RD method under different parameters, the effect of a small change (5\%) in the inertia is evaluated. By applying the RD method, the dynamic optimization process adequately demonstrates the distributed wellness of the control signal $x_{i}$ individually as shown in Figure 19. Signals in dashed lines $\left(x 1_{b}, x 2_{b}, x 3_{b}\right)$ show an increasing value in the signals, which mean that a bigger effort is required by the system. CoI Frequency response is shown Figure 20 where the primary control is dynamically optimized along the individual responses. RDb stands for the system with less inertia

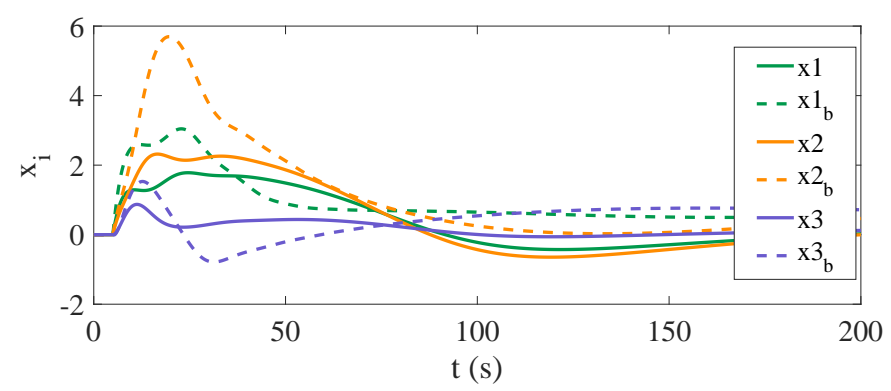

Figure 19: Wellness Variation

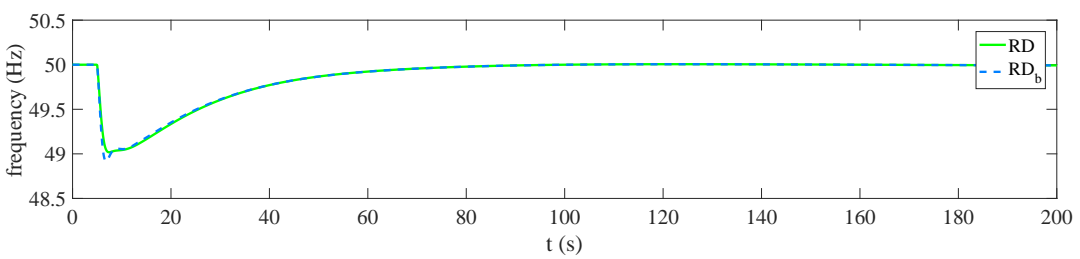

Figure 20: Frequency Response Variation

On the other hand, a static optimizer, such as SAA, finds the optimal solution following its objective function by the iteration process. A representation of this process is shown in Figure 21, where several results have been found. It starts with the less optimal (in violet), then other responses have a small overshoot (i.e. green). The final result (in magenta) accomplish the optimal primary control response and adjustment the parameters in the governors. Note for this setup it has been used one of the areas, the Swedish one in this case. 


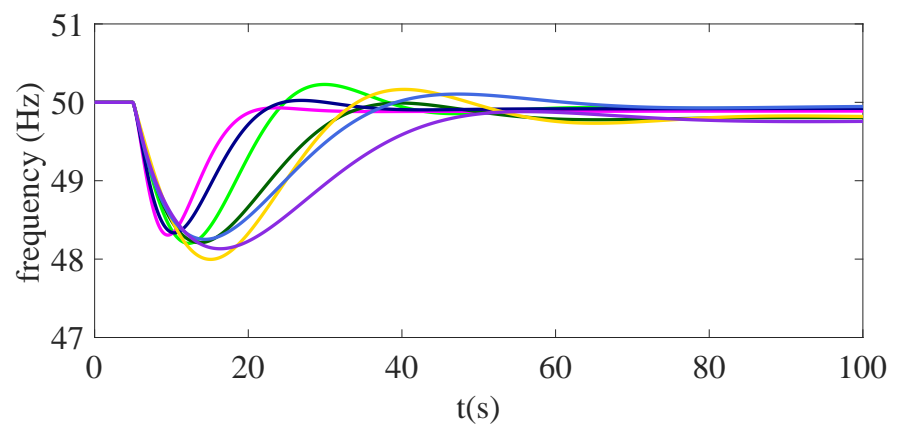

Figure 21: PID Tuned Process by Applying the SSA

\subsection{Nordic System}

The single-line diagram of the Nordic test system is shown in Figure 22. This system contains 32 high voltage buses, 20 synchronous generators with different types of generation, in four geographical identified areas. The North and External areas are hydro-dominated while the South and Central areas have a mixture of nuclear, thermal and coal power plants. Central area has the highest level consumption whereas the North area has the lowest level. The transmission system is designed for $400 \mathrm{kV}$ (19 buses) with some regional systems at $220 \mathrm{kV}$ (2 buses) and $130 \mathrm{kV}$ (11 buses). The details of the system, such as unit rating, line data, dynamic data, and loading conditions, are given in [53]. The simulation of this test system has been performed by the software DigSILENT ${ }^{\circledR}$.

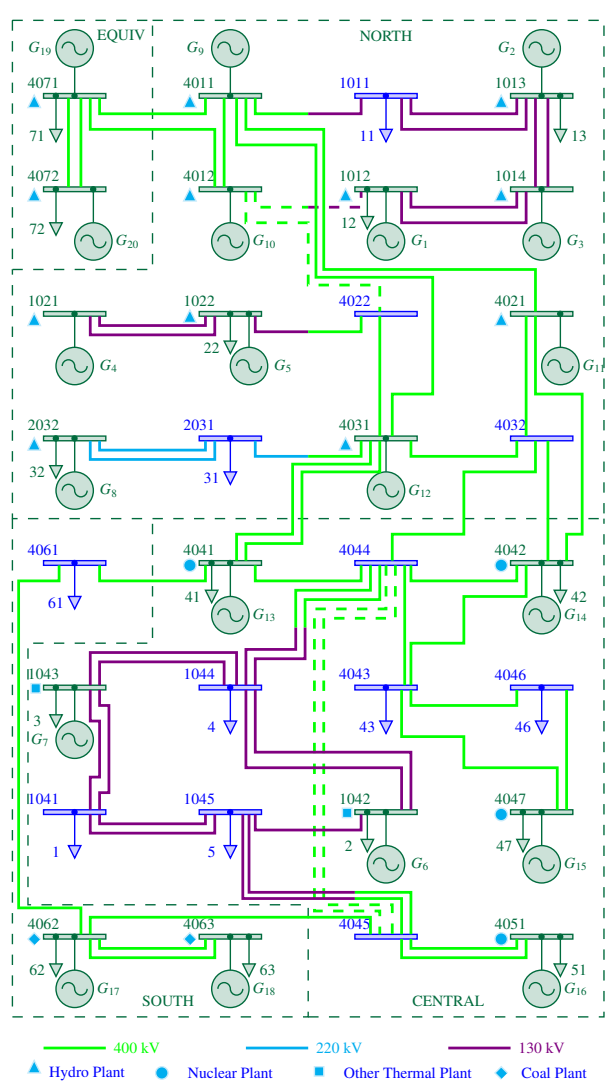

Figure 22: Nordic 32 Test System

Non-synchronous generation in this paper is defined as when the power is supplied or absorbed through power 
electronic converters (DC-AC) to/from the grid system. Voltage Source Converters (VSC) have been used for wind power turbines connections i.e., Full Rated Converters (FRC), Doubly-Fed Induction Generator (DFIG), and High Voltage Direct Current (HVDC) and Multi-terminal HVDC [54].

Table 8: Generator Replacement for Each Case

\begin{tabular}{|c|c|c|c|c|c|}
\hline Case & $C_{1}$ & $C_{2}$ & $C_{3}$ & $C_{4}$ & $C_{5}$ \\
\hline \hline Generator & $G_{7}$ & $G_{16}$ & $G_{17}$ & $G_{14}$ & $G_{6}$ \\
\hline Bus & 1043 & 4051 & 4062 & 4042 & 1042 \\
\hline Power (MW)\% & 1.2 & 5.4 & 9 & 13 & 15 \\
\hline
\end{tabular}

The impact of the integration of non-synchronous generation on the test system is analyzed by replacing some of the synchronous generators with non-synchronous generation with the same active and reactive power outputs. Note that the power outputs are fixed through the simulation. Case $1\left(C_{1}\right)$ to Case $5\left(C_{5}\right)$ represent the replacement of synchronous generation by the integration of non-synchronous generation gradually, in order to analyze different levels of power penetration. For example $C_{1}$ considers the replacement of one generation only, and $C_{2}$ considers the replacement of two generators including the one in $C_{1}$, and so on. It is assumed that the dispersed generation is connected to one established substation. These five scenarios are summarized in Table 8. However, only the cases where it has been found that significant changes occur are presented.

By the Figure 23, it can be seen the hydro-governors network is composed of the following associated machines: 1012, 1013, 1014, 1021, 1022, 4011, 4012, 4021, 2032, 4071, 4072 and 4031.

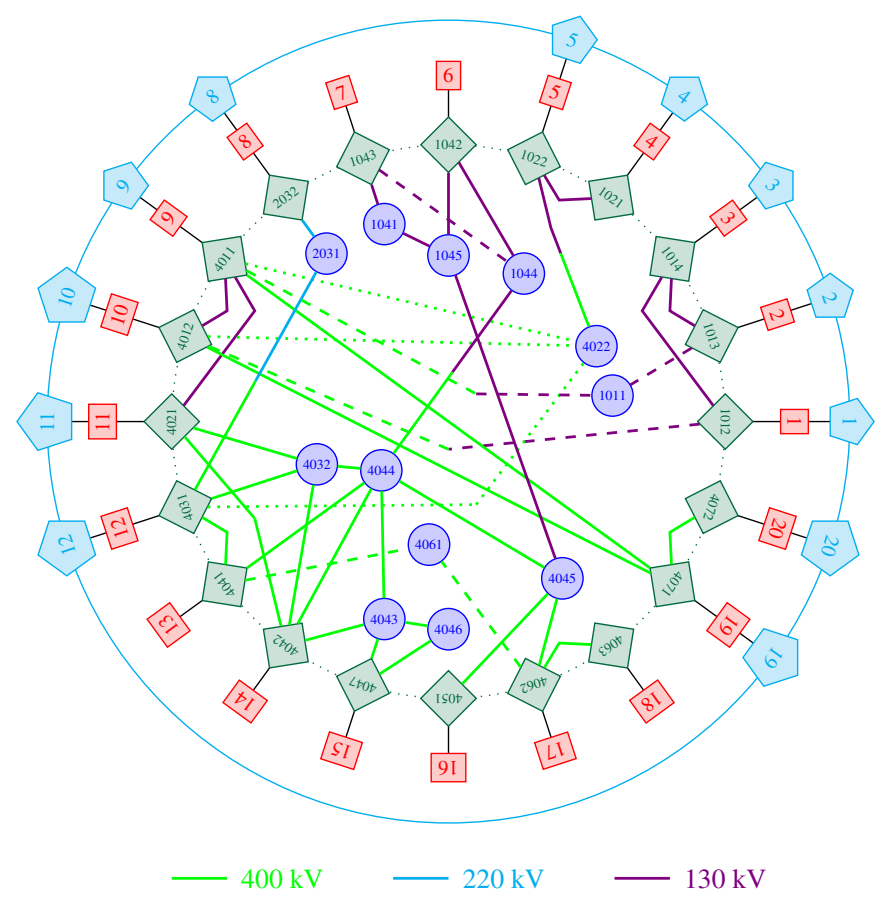

Figure 23: Nordic 32: Graph Representation

Figure 24 shows the system frequency response (in blue) and the RoCoF (in red) after disconnection of generator $G_{8}$ with $750 M W$. It can be seen that after the initial transient process (after approximately two seconds) the frequency falls at an almost constant rate of approximately $0.2 \mathrm{~Hz} / \mathrm{s}$ then the generation involved in the FCR starts to increase its production to compensate the loss. However, there remains a steady state error. 


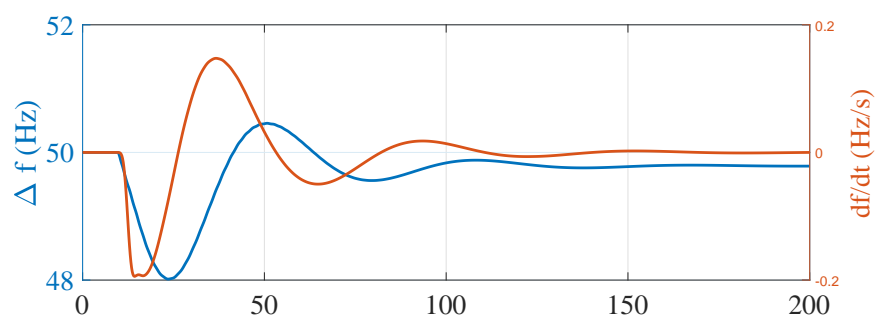

Figure 24: System Frequency Variation and ROCOF

In Table 9, some relevant metrics for this disturbance (base case $C_{0}$ ) are summarized.

Table 9: Relevant Metrics in Base Case

\begin{tabular}{|c|c|}
\hline Metric & Value \\
\hline \hline Maximum IFD [Hz] & 48.03 \\
\hline SSFD [Hz] & 0.2 \\
\hline Time to reach Max. IFD [s] & 14.63 \\
\hline
\end{tabular}

Cases stated in Table 8 are studied now. Figure 25 shows the time response of all cases when $G_{8}$ is tripped. As shown in the figure the more synchronous generators are replaced by wind power turbines, the larger dynamical frequency deviation becomes. A large frequency deviation may result in activating the protection systems of important components to be disconnected, and thereby leading to cascading failures in the system.

Table 10 shows the released kinetic energy by the remaining synchronous generators, and the ratio of the nonsynchronous generation power penetration with respect to the total generation for each case. Obviously, the released kinetic energy will decrease with higher non-synchronous generation penetration. The IFD, RoCoF and the SSFD of each case are also given in Table 10.

Table 10: Relevant Metrics for Each Case

\begin{tabular}{|c|c|c|c|c|}
\hline No. & Max. IFD [Hz] & Max. RoCoF [Hz/s] & SSFD & SH [GWs] \\
\hline \hline$C_{1}$ & 48.02 & 0.208 & 0.20 & 167 \\
\hline$C_{2}$ & 47.99 & 0.21 & 0.21 & 160 \\
\hline$C_{3}$ & 47.94 & 0.25 & 0.22 & 156 \\
\hline$C_{4}$ & 47.88 & 0.28 & 0.22 & 149 \\
\hline$C_{5}$ & 47.80 & 0.30 & 0.23 & 144 \\
\hline
\end{tabular}

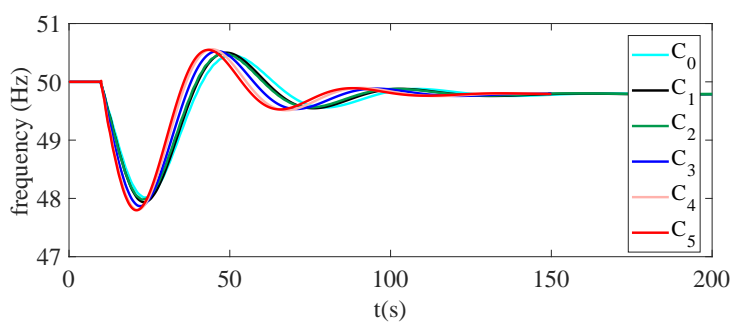

(a) Frequency Response of Each Case

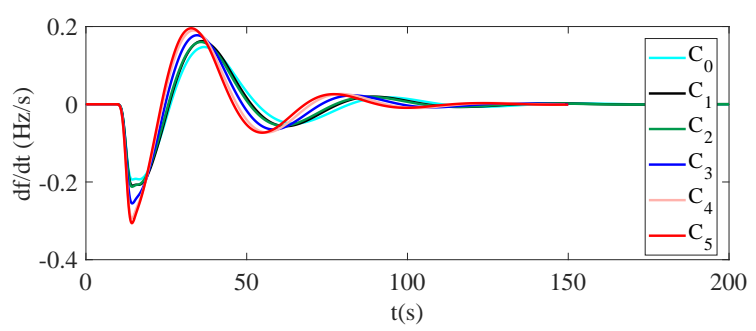

(b) RoCoF Response of Each Case

Figure 25: Frequency and RoCoF Response: Gradual Increasing of Non-Synchronous Generation 


\subsubsection{Replicator Dynamics and SAA Application}

The nominal frequency of the system is $50 \mathrm{~Hz}$ and is controlled by the speed governors of the hydro generators. The thermal generators of the Central and South areas are not involved in frequency control, and for the reasons explained in [45], constant mechanical torque is assumed for the machines of thermal plants. The model of the speed governor includes a simple power measurement, a PI control and a servomotor represented by a first-order system with a time constant of $0.2 \mathrm{~s}$ and non-windup limits on $g$ which represents the gate opening.

The speed governor gives the gate opening to the hydro turbine model, which is represented by a simple lossless model with a water time constant $T_{\omega}$ of $1 \mathrm{~s}$. In this model $q$ represents the water flow, $h$ the head, and $P_{m}$ the mechanical power. Figure 26 shows the non-linear model of the turbine used. Figure 23 shows the graph topology representation and the governors network control system.

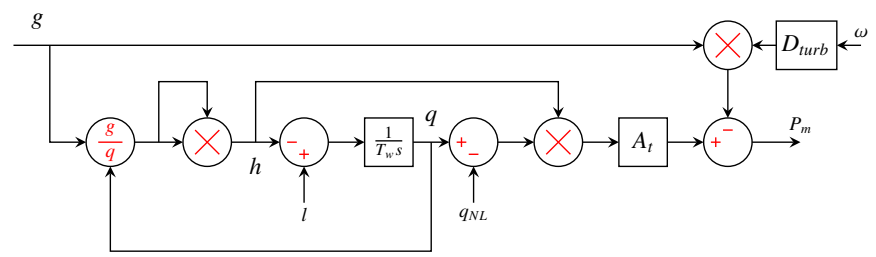

Figure 26: Simplified Nonlinear Turbine Model

The optimization SAA method and the RD method are added to the Nordic test system as before. Figure 27 shows the comparison responses between the Case 5, the RD application and RD-SAA combination in green, orange and blue respectively. By applying the SAA and RD methods, at the same time is obtained the global optimal parameters for the optimal parameters for the optimal performance of the frequency response of the system. However, by the application of the mere RD method a close response to the optimal has been reached. The reason that the SAA obtained the global parameters, and therefore a better response is due to the multiple iterations and the conditions given by the objective function which includes different metrics in the signal. However, the computational effort for obtaining those optimal parameters is quite high, compared to the RD methods which does it dynamically and does not require to do run multiple times. Both methods accomplish their objective, the improvement of the frequency response and the counteracting of a large penetration of non-synchronous generation.

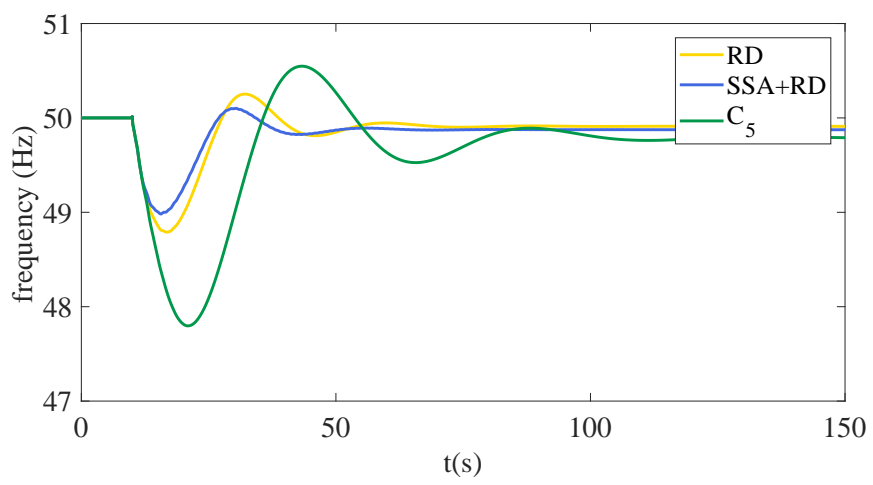

Figure 27: Frequency Response

Table 11 presents the performance metrics by the two methods applied to the system. The response of the system has been improved. By comparing the time reaction, which is calculated as the difference between the nadir-time and the settling time, an approximate 4 seconds difference between the SAA and RD methods is obtained. 
Table 11: Optimal Parameters vs RD

\begin{tabular}{|c|c|}
\hline Parameter & CoI \\
\hline \hline$t_{s}-s a a$ & 57.21 \\
\hline$t_{s}-r d$ & 64.23 \\
\hline$o_{v}-s a a$ & 0.45 \\
\hline$o_{v}-r d$ & 0.68 \\
\hline nadir $-s a a$ & 48.98 \\
\hline nadir $^{-r d}$ & 48.79 \\
\hline nadir $_{t}-s a a$ & 15.62 \\
\hline nadi $_{t}-r d$ & 16.52 \\
\hline
\end{tabular}

\section{Discussion}

In general, SAA approach and meta-heuristic optimization methods rely on simulating the system and comparing the objective function against the response by iteration. SAA certainly provides optimal parameters for the test systems proposed, the hydro-governors enhancement and improving the frequency response in the system. However, one of the main drawbacks it has is the high computational cost that it requires, especially when it is required to be implemented in large complex systems. Additionally, if any of the other parameters in the system changes, it is needed to execute the optimization process again. On the other hand, the RD, as a supplementary network control, and as a dynamic optimizer, it is capable of distribute dynamic and optimal actions through the network control systems. However, the use of $\mathrm{RD}$ is restricted to just one only distributive action, the derivative action in this case. This is based on the boundary and barrier constraints of the protocol. Also, it requires a network linked topology for receiving the outputs from the power system and for distributing the respective action. Both methods, the optimized derivative control based on SAA and the RD based on the RoCoF measurement, are suitable for the hydro-governors enhancement, improving the frequency response and counteracting the frequency events presented in the system.

\section{Conclusions}

In this paper, two approaches are given in order to improve the hydro governors reaction in order to improve the primary control and, counteract a frequency event in power systems caused by the inertia reduction due to the non-synchronous generation integration. The first approach is based on the application of a meta-heuristic optimal method, the SS algorithm, and a dynamic resource allocation method, the RD. By adding the optimal derivative control action obtained from the SA algorithm, and the addition of the distributive supplementary action based on the RoCoF measurement showed the improvement of the time-reaction of the hydro-governors, thereby, improving the frequency response.

For validating the methods, two systems have been evaluated by the two methods presented. The first test system is based on a common modeling framework for frequency studies which represents the aggregated model of the Nordic countries. The second system is the Nordic 32 test system where is a large gradually increasing of non-synchronous generation. The simulation results confirm the validation of the method improving the frequency response in the test system through the hydro-governors network.

In practice, the addition of an extra control variable can be easily electronically implemented, and considering the integration of RD type of controller, it may not require any major replacement of the existing infrastructure in the AGC architecture.

During the design of the RD application method, one important observation was found: since a machine with bigger inertia reacts less to a negative disturbance, the control law is then distributed based on the machine's effort .

Future research directions include, but are not limited to the incorporation of more optimization algorithms, the exploration of other network control systems protocols. In the latest in particular, RD method requires future development for the application of multiple distributed signals in multi-input multi-output systems in order to guarantee a multi-welfare assignment. Moreover, a further study and analysis should incorporate the variation of several parameters in the system. 


\section{Appendix A. SAA Fundamentals}

\section{Appendix A.1. State Generation Function}

State Generation Function is a function that generates a random solution in solution space. Normally, Gaussian Distribution is employed to generate random solution. The State Generation Function must guarantee that random solutions can cover the whole solution space.

\section{Appendix A.2. State Acceptance Function}

State Acceptance Function is a function that generate the probability to accept a new state or not. According to thermodynamics, at temperature $T$, the probability of an increase in energy of $\Delta E$ is:

$$
p(\Delta E)=e^{-\frac{\Delta E}{k_{B} T}}
$$

where $k_{B}$ is the Boltzmann constant.

Similarly, in Simulated Annealing Algorithm, we define the State Acceptance Function $S$ in the form below:

$$
S= \begin{cases}e^{-\frac{\Delta J}{T}}, & \Delta J>0 \\ 1, & \Delta J \leq 0\end{cases}
$$

where $\Delta J$ is the difference of new state's Objective Function value and last state's Objective Function value, and $T$ is the 'temperature' of last state.

\section{Appendix A.3. Temperature Renew Function}

To ensure the convergence to globally optimal solution of the algorithm, the Temperature Renew Function must match the State Generation Function. With non-homogeneous chain, which can guarantee asymptotically convergence, Temperature Renew Function is chosen as below,

$$
T_{k_{B}}=\frac{c}{\log \left(k_{B}+1\right)}
$$

where $c$ is the depth of the deepest local minimum, $k_{B}$ is a constant.

But the cooling of temperature will be very slow if the function above is chosen. In practice, the Temperature Renew Function as below is always employed, which can solve the speed problem of cooling process:

$$
T_{k_{B}}=\frac{T}{1+\vartheta}
$$

where $\vartheta$ is a positive real number.

\section{Appendix A.4. Length of Markov Chain $L_{m}$}

The length of Markov chain corresponds the maximal times of iteration in Simulated Annealing Algorithm.

\section{Appendix A.5. Stop Criterion}

To stop the iteration, we need to set a final value of temperature $T_{f}$ and Tolerance Value $V_{T}$. If the change of the Objective Function is within the Tolerance Value $V_{T}$, it means the change of Objective Function is small enough and we can consider it has already reach the optimal solution. Thus, if the temperature $T$ has reached $T_{f}$ or the change of Objective Function is within our Tolerance Value $V_{T}$ for several steps, then the iteration will be stopped and return the final accepted solutions as the optimal solutions. 


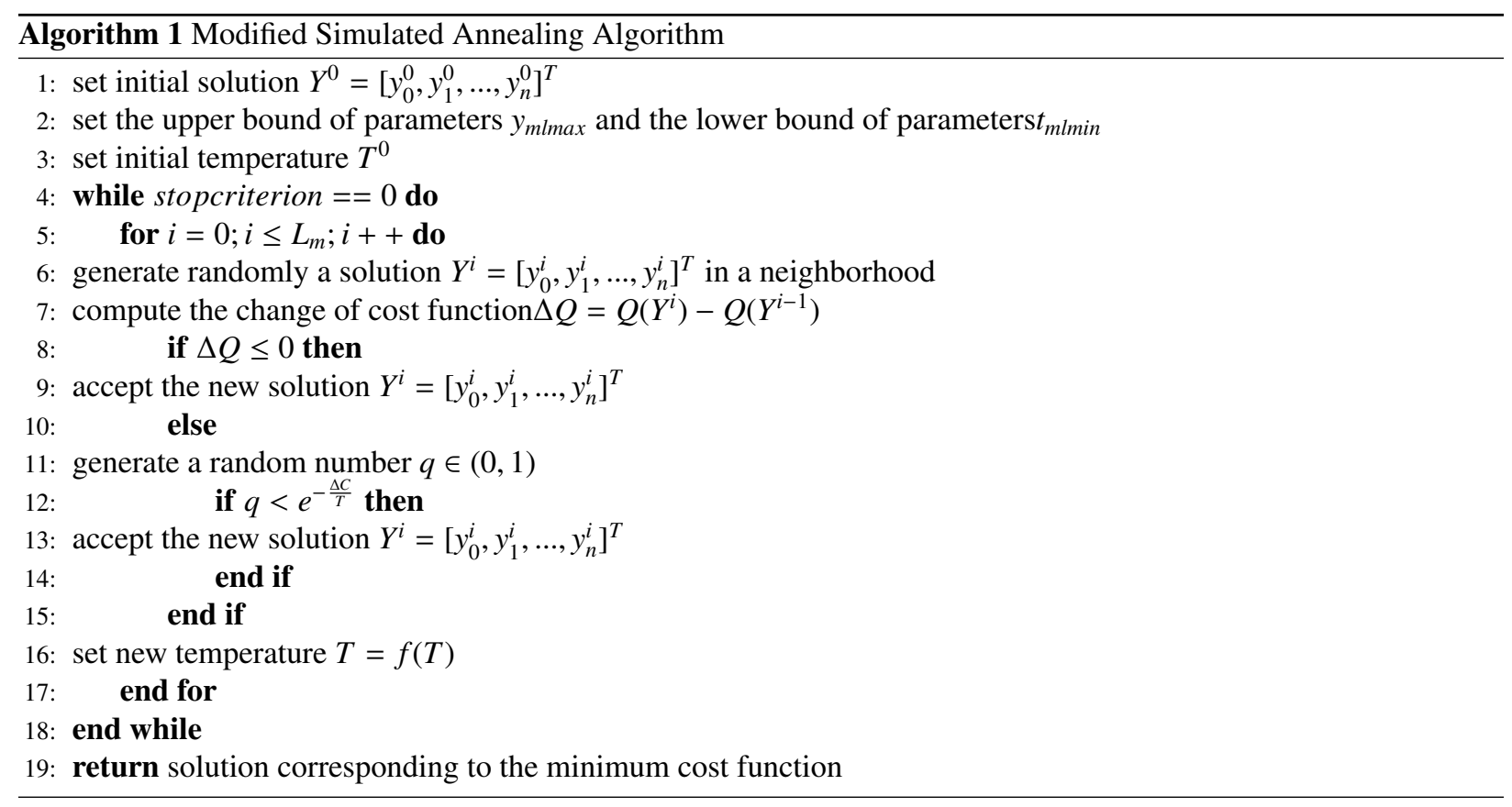

\section{Appendix B. SAA Algorithm}

\section{Appendix C. Routh-Hurwitz Stability Proof}

From $G(s)$ the terms of the characteristic polynomial are obtained and Routh-Hurwitz's criterion is applied to establish the stable boundaries. Let $a_{i}$ be characteristic polynomial coefficients and, let $b_{i}, c_{i}, d_{i}$ and $e_{i}$ be RouthHurwitz's coefficients, then, the set $\Omega_{s}$ is defined by follow optimization problem:

$$
\Omega^{s}=\forall\left(k_{i}^{p}, k_{i}^{i}\right):\left\{\begin{aligned}
\max & u: k_{i}^{p}+k_{i}^{i} \\
\text { subject to } & g_{1}: a_{5}>0 \\
& g_{2}: a_{4}\left(k_{i}^{p}\right)>0 \\
& g_{3}: a_{3}\left(k_{i}^{p}, k_{i}^{i}\right)>0 \\
& g_{4}: a_{2}\left(k_{i}^{p}, k_{i}^{i}\right)>0 \\
& g_{5}: a_{1}\left(k_{i}^{p}, k_{i}^{i}\right)>0 \\
& g_{6}: a_{0}\left(k_{i}^{i}\right)>0 \\
& g_{7}: b_{1}\left(k_{i}^{p}, k_{i}^{i}\right)>0 \\
& g_{8}: c_{1}\left(k_{i}^{p}, k_{i}^{i}\right)>0 \\
& g_{9}: d_{0}\left(k_{i}^{p}, k_{i}^{i}\right)>0 \\
& g_{10}: e_{0}\left(k_{i}^{i}\right)>0 \\
& g_{11}: k_{i}^{p}>0 \\
& g_{12}: k_{i}^{i}>0
\end{aligned}\right.
$$




\section{Appendix D. Passivity Stability Proof}

In order to show that the origin of the feedback interconnected system (13) is stable, first we need to prove that Power System $\Sigma^{p}$, with input $\mathbf{u}_{1}=\left[x_{1}, \ldots, x_{n_{g}}\right]^{\top}$, and output $\mathbf{y}_{1}=\left[-f_{1}+c, \ldots,-f_{n_{g}}+c\right]^{\top}$, is strictly passive. Then, we need to prove that Distributed Control via RD $\Sigma^{c}$, with input $\mathbf{u}_{2}=\left[-f_{1}+c, \ldots,-f_{n_{g}}+c\right]^{\top}$, and output $\mathbf{y}_{2}=\left[-x_{1}, \ldots,-x_{n_{g}}\right]^{\top}$, is lossless.

For $\Sigma^{p}, \Sigma^{p}$ is a linear system, then the system is strictly passive if

$$
G(j \omega)+G^{*}(j \omega)>0, \forall \omega \in \mathbb{R}
$$

Therefore, all poles of $G(s)$ must have strictly negative real part. Now,

$$
G(s)=\mathbf{C}_{i_{0}}\left(s \mathbf{I}-\mathbf{A}_{\mathbf{i}_{0}}\right)^{-1} \hat{\mathbf{B}}_{\mathbf{i}_{0}}+\mathbf{D}_{\mathbf{i}_{0}}
$$

Analyzing only the input $\mathbf{u}_{1}, \omega_{\text {ref }}=0$ and $P_{i}^{e}=0$, then

$$
\begin{gathered}
\hat{\mathbf{B}}_{i_{0}}=\left[\begin{array}{c}
0 \\
0 \\
\frac{k_{i}^{t}}{T_{i}^{p}} \\
0 \\
0
\end{array}\right] \\
\mathbf{C}_{i_{0}}=\left[\begin{array}{ccccc}
1-\frac{D_{i}}{M_{i}} & \frac{1}{M_{i}} & 0 & 0 & 0
\end{array}\right] \\
\mathbf{D}_{i_{0}}=\left[\begin{array}{ll}
0 & 0
\end{array}\right]
\end{gathered}
$$

Refer to Appendix Appendix C.

Let $\eta_{i}^{i}(\cdot)$ be a function with domain in the ordinate of $\Omega_{s}$ and range on the abscissa of $\Omega_{s}$. Now, let 0 y $\eta_{i}^{p}$ will be the infimum and the supremum in the domain of $\eta_{i}^{i}(\cdot)$. It says that $\left(k_{i}^{p *}, k_{i}^{i^{*}}\right)$ is stable if $0<k_{i}^{i^{*}}<\eta_{i}^{i}\left(k_{i}^{p *}\right)$ and $0<k_{i}^{p *}<\eta_{i}^{p}$, satisfying with Routh-Hurwitz's criterion. So, all poles of $G(s)$ have a strictly real negative part and the system $\Sigma^{p}$ is strictly passive.

Now, for $\Sigma^{c}$, we choose the positive definite storage function $V^{c}(x)$ :

$$
V^{c}(x)=-\frac{1}{\beta} \sum_{i=1}^{N} \omega_{C o I} \ln \left(\frac{x_{i}+\omega_{C o I}}{\omega_{C o I}}\right)
$$

The derivative of $V^{c}(x)$ along the trajectories of $\Sigma^{c}$ is given by (D.7).

$$
\begin{aligned}
\dot{V}^{c}(x) & =-\frac{1}{\beta} \sum_{i=1}^{N} \omega_{\text {CoI }}\left[\frac{\dot{x}_{i}}{x_{i}+\omega_{\text {CoI }}}\right] \\
& =-\sum_{i=1}^{N} \omega_{C o I}\left[f_{i}-\sum_{j=1}^{N} f_{j}\left(x_{j}+\omega_{C o I}\right)\right] \\
& =-\sum_{i=1}^{N} \omega_{C o I} f_{i}+\sum_{i=1}^{N} f_{i}\left(x_{i}+\omega_{C o I}\right)
\end{aligned}
$$

So we have that

$$
\mathbf{u}_{2}^{\top} \mathbf{y}_{2}=\sum_{i=1}^{N}\left(f_{i}-c\right) x_{i}=\dot{V}^{c}(x)
$$

Then, let us prove that $\Sigma^{c}$ is lossless. 


\section{References}

[1] T. Brown, “Transmission network loading in Europe with high shares of renewables," IET Renewable Power Generation, vol. 9, no. 1, pp. 57-65, 2015.

[2] W. Winter, K. Elkington, G. Bareux, and J. Kostevc, "Pushing the Limits: Europe's New Grid: Innovative Tools to Combat Transmission Bottlenecks and Reduced Inertia," IEEE Power and Energy Magazine, vol. 13, pp. 60-74, Jan. 2015.

[3] M. Imhof and G. Andersson, "Power system stability control using Voltage Source Converter based HVDC in power systems with a high penetration of Renewables," in 2014 Power Systems Computation Conference, pp. 1-7, Aug. 2014.

[4] E. Rakhshani, D. Remon, A. M. Cantarellas, and P. Rodriguez, "Analysis of derivative control based virtual inertia in multi-area high-voltage direct current interconnected power systems," Transmission Distribution IET Generation, vol. 10, no. 6, pp. 1458-1469, 2016.

[5] N. W. Miller, "Keeping It Together: Transient Stability in a World of Wind and Solar Generation," IEEE Power and Energy Magazine, vol. 13, pp. 31-39, Nov. 2015.

[6] P. Kundur, J. Paserba, V. Ajjarapu, G. Andersson, A. Bose, C. Canizares, N. Hatziargyriou, D. Hill, A. Stankovic, C. Taylor, T. V. Cutsem, and V. Vittal, "Definition and classification of power system stability IEEE/CIGRE joint task force on stability terms and definitions," IEEE Transactions on Power Systems, vol. 19, pp. 1387-1401, Aug. 2004.

[7] "Hydraulic turbine and turbine control models for system dynamic studies," IEEE Transactions on Power Systems, vol. 7, pp. 167-179, Feb. 1992.

[8] N. Kishor, R. P. Saini, and S. P. Singh, "A review on hydropower plant models and control," Renewable and Sustainable Energy Reviews, vol. 11, pp. 776-796, June 2007.

[9] J. Culberg, M. Negnevitsky, and K. M. Muttaqi, "Hydro-turbine governor control: theory, techniques and limitations," 2006.

[10] Y. C. Choo, K. M. Muttaqi, and M. Negnevitsky, "Modelling of hydraulic turbine for dynamic studies and performance analysis," in 2007 Australasian Universities Power Engineering Conference, pp. 1-6, Dec. 2007.

[11] L. Saarinen, P. Norrlund, U. Lundin, E. Agneholm, and A. Westberg, "Full-scale test and modelling of the frequency control dynamics of the Nordic power system," in 2016 IEEE Power and Energy Society General Meeting (PESGM), pp. 1-5, July 2016.

[12] J. Xi, H. R. Chamorro, J. Persson, A. Westberg, D. Wall, and M. Ghandhari, "On the influence of the backlash governor settings on the frequency response in power systems," in 2016 IEEE PES Asia-Pacific Power and Energy Engineering Conference (APPEEC), pp. 732-737, Oct. 2016.

[13] J. Culberg, M. Negnevitsky, and K. M. Muttaqi, "Fuzzy inference system controller for hydro turbine-generator system," in 2007 Australasian Universities Power Engineering Conference, pp. 1-6, Dec. 2007.

[14] X. Yu, F. Yang, Y. Huang, and H. Nan, "Fuzzy immune sliding mode control based hydro turbine governor," in Third International Conference on Natural Computation (ICNC 2007), vol. 1, pp. 171-176, Aug. 2007.

[15] A. Sami, M. B. Kadri, N. Aziz, and Z. Pirwani, "Design simulation of fuzzy PID for hydro power plant," in 2016 Sixth International Conference on Innovative Computing Technology (INTECH), pp. 683-687, Aug. 2016.

[16] S. Liu, D. Li, and C.-e. Huang, "Nonlinear robust control with high gain observer for governor of hydro-turbine generator sets," in Proceedings of the 10th World Congress on Intelligent Control and Automation, pp. 2752-2757, July 2012.

[17] D. Qing and Y. Xiangwu, "Evaluate the Effect of Hydro Turbine Governor Decentralized Control by Transient Energy," in 2006 Chinese Control Conference, pp. 1655-1659, Aug. 2006.

[18] F. Yang, H. Lei, Y. Sun, W. Lin, and T. Shen, "Control of hydraulic turbine generators using exact feedback linearization," in IEEE ICCA 2010, pp. 1372-1378, June 2010.

[19] I. Eker, "Governors for hydro-turbine speed control in power generation: a SIMO robust design approach," Energy Conversion and Management, vol. 45, pp. 2207-2221, Aug. 2004.

[20] R. Sebastian and J. Quesada, "Modeling and simulation of an isolated wind Hydro Power System," in IECON 2016 - 42nd Annual Conference of the IEEE Industrial Electronics Society, pp. 4169-4174, Oct. 2016.

[21] J. Zou, M. Pipattanasomporn, S. Rahman, and X. Lai, "A Frequency Regulation Framework for Hydro Plants to Mitigate Wind Penetration Challenges," IEEE Transactions on Sustainable Energy, vol. 7, pp. 1583-1591, Oct. 2016.

[22] R. T. K and R. P, "Frequency control of wind penetrated hydro-dominated power system," in 2015 International Conference on Technological Advancements in Power and Energy (TAP Energy), Jun. 2015, pp. 316-321.

[23] J. Dong, A. B. Attya, and O. Anaya-Lara, "Frequency stability analysis in low frequency AC systems for renewables power transmission," in 2017 6th International Conference on Clean Electrical Power (ICCEP), Jun. 2017, pp. 275-279.

[24] E. Rakhshani and P. Rodriguez, "Inertia Emulation in AC/DC Interconnected Power Systems Using Derivative Technique Considering Frequency Measurement Effects," in IEEE Transactions on Power Systems, vol. 32, no. 5, pp. 3338-3351, Sept. 2017.

[25] A. Moawwad, E. El-Saadany and M. S. El Moursi, "Dynamic Security-Constrained Automatic Generation Control (AGC) of Integrated AC/DC Power Networks," in IEEE Transactions on Power Systems, vol. PP, no. 99, pp. 1-1.

[26] M. Guan, W. Pan, J. Zhang, Q. Hao, J. Cheng and X. Zheng, "Synchronous Generator Emulation Control Strategy for Voltage Source Converter (VSC) Stations," in IEEE Transactions on Power Systems, vol. 30, no. 6, pp. 3093-3101, Nov. 2015.

[27] D. Ochoa and S. Martinez, "Fast-Frequency Response Provided by DFIG-Wind Turbines and its Impact on the Grid," in IEEE Transactions on Power Systems, vol. 32, no. 5, pp. 4002-4011, Sept. 2017.

[28] M. Persson and P. Chen, "Frequency control by variable speed wind turbines in islanded power systems with various generation mix," IET Renewable Power Generation, vol. 11, no. 8, pp. 1101-1109, 2017.

[29] X. Liu, Y. Zhang, and K. Y. Lee, "Coordinated Distributed MPC for Load Frequency Control of Power System With Wind Farms," IEEE Transactions on Industrial Electronics, vol. 64, no. 6, pp. 5140-5150, Jun. 2017.

[30] F. L. Lewis, Cooperative control of multi-agent systems: optimal and adaptive design approaches. Amsterdam: Springer, 2014. OCLC: 891508597

[31] A. W. Colombo, S. Karnouskos, O. Kaynak, Y. Shi, and S. Yin, "Industrial Cyberphysical Systems: A Backbone of the Fourth Industrial Revolution,” IEEE Industrial Electronics Magazine, vol. 11, pp. 6-16, Mar. 2017. 
[32] Z. Li, C. Zang, P. Zeng, H. Yu, and H. Li, "MAS based distributed automatic generation control for cyber-physical microgrid system," IEEE/CAA Journal of Automatica Sinica, vol. 3, pp. 78-89, Jan. 2016.

[33] H. R. Chamorro and N. L. Diaz, "Hierarchical power flow control in low voltage microgrids," in 2013 North American Power Symposium (NAPS), pp. 1-5, Sept. 2013.

[34] V. Nasirian, Q. Shafiee, J. M. Guerrero, F. L. Lewis, and A. Davoudi, "Droop-Free Distributed Control for AC Microgrids," IEEE Transactions on Power Electronics, vol. 31, pp. 1600-1617, Feb. 2016.

[35] F. Dorfler, M. R. Jovanovic, M. Chertkov, and F. Bullo, "Sparsity-Promoting Optimal Wide-Area Control of Power Networks," IEEE Transactions on Power Systems, vol. 29, pp. 2281-2291, Sept. 2014.

[36] S. C. Muller, U. Hager, and C. Rehtanz, "A Multiagent System for Adaptive Power Flow Control in Electrical Transmission Systems," IEEE Transactions on Industrial Informatics, vol. 10, pp. 2290-2299, Nov. 2014.

[37] H. R. Chamorro, A. C. Sanchez, A. Øverjordet, F. Jimenez, F. Gonzalez-Longatt and V. K. Sood, "Distributed synthetic inertia control in power systems," 2017 International Conference on ENERGY and ENVIRONMENT (CIEM), Bucharest, 2017, pp. 74-78.

[38] A. Pantoja and N. Quijano, "A Population Dynamics Approach for the Dispatch of Distributed Generators," IEEE Transactions on Industrial Electronics, vol. 58, pp. 4559-4567, Oct. 2011.

[39] G. Chen, F. L. Lewis, E. N. Feng, and Y. Song, "Distributed Optimal Active Power Control of Multiple Generation Systems," IEEE Transactions on Industrial Electronics, vol. 62, pp. 7079-7090, Nov. 2015.

[40] M. Wooldridge, An Introduction to MultiAgent Systems, 2nd ed. Chichester, U.K: Wiley, Jun. 2009.

[41] M. Andreasson, D. V. Dimarogonas, H. Sandberg, and K. H. Johansson, "Distributed Control of Networked Dynamical Systems: Static Feedback, Integral Action and Consensus," IEEE Transactions on Automatic Control, vol. 59, no. 7, pp. 1750-1764, Jul. 2014.

[42] P. D. Taylor and L. B. Jonker, "Evolutionary stable strategies and game dynamics," Mathematical Biosciences, vol. 40, pp. 145-156, July 1978.

[43] A. Ovalle, A. Hably, S. Bacha, G. Ramos, and J. M. Hossain, "Escort Evolutionary Game Dynamics Approach for Integral Load Management of Electric Vehicle Fleets," IEEE Transactions on Industrial Electronics, vol. 64, pp. 1358-1369, Feb. 2017.

[44] J. W. Weibull, Evolutionary game theory. Cambridge, Mass. [u.a.: MIT Press, 2004. OCLC: 255505178.

[45] K. Luo and W. Shi, "Distributed coordination method of microgrid economic operation optimization based on multi-agent system," in 2014 International Conference on Power System Technology, pp. 3135-3140, Oct. 2014.

[46] W. H. Sandholm, "Population games and evolutionary dynamics," 2010.

[47] J. Hofbauer and W. H. Sandholm, "Stable games and their dynamics," Journal of Economic Theory, vol. 144, no. 4, pp. 1665-1693, 2009.

[48] K. Iewaki and T. Kanazawa, "Evolutionary dynamics for multicriteria games generated by p-norm-based pairwise proportional imitation," in 2016 IEEE International Conference on Systems, Man, and Cybernetics (SMC), pp. 002744-002749, Oct. 2016.

[49] S. Kirkpatrick, C. D. Gelatt, and M. P. Vecchi, “Optimization by Simulated Annealing,” Science, vol. 220, no. 4598, pp. 671-680, 1983.

[50] N. S. Nise, Control Systems Engineering, 7th ed. Hoboken, NJ: Wiley, Feb. 2015.

[51] H. K. Khalil, "Noninear systems," 1996.

[52] N. Quijano, C. Ocampo-Martinez, J. Barreiro-Gomez, G. Obando, A. Pantoja, and E. Mojica-Nava, "The role of population games and evolutionary dynamics in distributed control systems: The advantages of evolutionary game theory," IEEE Control Systems, vol. 37, pp. 70-97, Feb 2017.

[53] C. T. Force, "Long term dynamics phase II final report," CIGRE, Mar.1995.

[54] H. R. Chamorro, C. A. Ordonez, J. C. Peng, and M. Ghandhari, "Non-synchronous generation impact on power systems coherency", Transmission Distribution IET Generation, vol. 10, no. 10, pp. 2443-2453, 2016. 